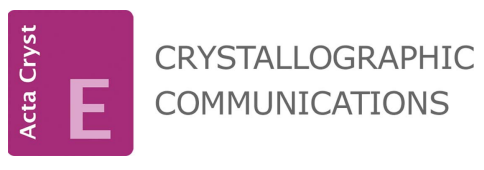

ISSN 2056-9890

Received 22 April 2020

Accepted 22 May 2020

Edited by J. T. Mague, Tulane University, USA

Keywords: crystal structure; platinum(II) complexes; eugenol; pyridine derivatives; cytotoxicity.

CCDC references: 2005230; 2005229; 2005228

Supporting information: this article has supporting information at journals.iucr.org/e

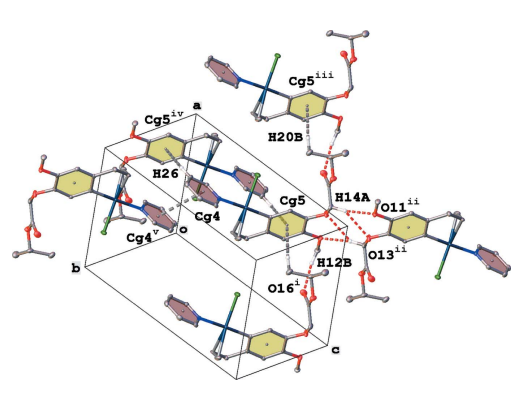

OPEN $\odot$ ACCESS

\section{Crystal structures of three platinacyclic complexes bearing isopropyl eugenoxyacetate and pyridine derivatives}

\author{
Nguyen Thi Thanh Chi, ${ }^{a}$ Pham Van Thong ${ }^{a}$ and Luc Van Meervelt ${ }^{b *}$ \\ a'Department of Chemistry, Hanoi National University of Education, 136 Xuan Thuy, Cau Giay, Hanoi, Vietnam, and \\ ${ }^{b}$ Department of Chemistry, KU Leuven, Biomolecular Architecture, Celestijnenlaan 200F, Leuven (Heverlee), B-3001, \\ Belgium. *Correspondence e-mail: Luc.VanMeervelt@kuleuven.be
}

Three new platinum(II) complexes bearing a eugenol and a pyridine derivative, namely $\quad\left(\eta^{2}\right.$-2-allyl-4-methoxy-5-\{[(propan-2-yloxy)carbonyl]methoxy\}phenyl$\left.\kappa C^{1}\right)$ chlorido(pyridine- $\left.\kappa N\right)$ platinum(II), $\left[\mathrm{Pt}\left(\mathrm{C}_{15} \mathrm{H}_{19} \mathrm{O}_{4}\right) \mathrm{Cl}\left(\mathrm{C}_{5} \mathrm{H}_{5} \mathrm{~N}\right)\right],(\mathbf{I}),\left(\eta^{2}-2-\right.$ allyl-4-methoxy-5-\{[(propan-2-yloxy)carbonyl $]$ methoxy\}phenyl- $\left.\kappa C^{1}\right)$ chlorido(4methylpyridine- $\kappa N)$ platinum(II), $\left[\mathrm{Pt}\left(\mathrm{C}_{15} \mathrm{H}_{19} \mathrm{O}_{4}\right) \mathrm{Cl}\left(\mathrm{C}_{6} \mathrm{H}_{7} \mathrm{~N}\right)\right],(\mathbf{I I})$, and $\left(\eta^{2}-2-\right.$ allyl-4-methoxy-5-\{[(propan-2-yloxy)carbonyl]methoxy\}phenyl- $\left.\kappa C^{1}\right)$ chlorido(pyridine-4-carboxylic acid- $\kappa N)$ platinum(II), $\quad\left[\mathrm{Pt}\left(\mathrm{C}_{15} \mathrm{H}_{19} \mathrm{O}_{4}\right) \mathrm{Cl}\left(\mathrm{C}_{6} \mathrm{H}_{5} \mathrm{NO}_{2}\right)\right]$, (III), have been synthesized and further characterized by single-crystal X-ray diffraction. The $\mathrm{Pt}^{\mathrm{II}}$ atoms exhibit the usual distorted square-planar coordination and are surrounded by one $\mathrm{Cl}$ atom, one $\mathrm{N}$ atom, and a $\mathrm{C}$ atom and $\mathrm{C}=\mathrm{C}$ double bond of the eugenol ligand. The donor $\mathrm{N}$ atom of the pyridine ligand occupies a cis position with respect to the double bond. Complexes (I) and (II) crystallize isomorphously in space group $P \overline{1}$ and display a similar crystal packing characterized by $\mathrm{C}-\mathrm{H} \cdots \mathrm{O}$ hydrogen bonding, $\mathrm{C}-\mathrm{H} \cdots \pi$ and $\pi-\pi$ interactions. However, the presence of the additional methyl group in the 4-methylpyridine ligand in (II) disturbs the $\pi-\pi$ interactions. The crystal packing of (III) is characterized by $\mathrm{O}-\mathrm{H} \cdots \mathrm{O}$ hydrogen bonding, resulting in the formation of chains of molecules connected in a head-to-tail fashion and running in the [101] direction. The $\mathrm{IC}_{50}$ values for the HepG2 and KB cell lines are 150.9, 122.3 $\mu M$ for (I) and 138.9, 93.2 $\mu M$ for (II), respectively.

\section{Chemical context}

Although platinum-based drugs have dominated the treatment of various cancers by chemical agents, the research on new platinum(ll) complexes for the purpose of medical application is still attractive for the worldwide scientific society (Johnstone et al., 2016). Recently, numerous platinum(11) complexes bearing alkene and pyridine derivatives have been synthesized and tested for their anti-cancer activities (Bigioni et al., 2000; Da et al., 2012, 2015; Chi et al., 2017, 2018; Cucciolito et al., 2018; Dodoff et al., 2012). Nevertheless, crystal data for these complexes are limited, some examples being the crystal structures of [PtCl(eugenol- $1 H$ )(pyridine)], [PtCl(eugenol-1H)(4-methylpyridine)] (Chi et al., 2018) and trans- $\left[\mathrm{PtCl}_{2}\left(\mathrm{C}_{2} \mathrm{H}_{4}\right)(N\right.$-3-pyridinylmethanesulfonamide)] (Dodoff et al., 2012).

In this paper, the crystal structures of three mononuclear platinacyclic complexes namely, ( $\eta^{2}$-2-allyl-4-methoxy-5$\left\{[(\right.$ propan-2-yloxy)carbonyl $]$ methoxy $\}$ phenyl- $\left.\kappa C^{1}\right)$ chlorido(pyridine- $\kappa N)$ platinum(II), $\quad\left[\mathrm{Pt}\left(\mathrm{C}_{15} \mathrm{H}_{19} \mathrm{O}_{4}\right) \mathrm{Cl}\left(\mathrm{C}_{5} \mathrm{H}_{5} \mathrm{~N}\right)\right], \quad(\mathbf{I})$, $\left(\eta^{2}\right.$-2-allyl-4-methoxy-5-\{[(propan-2-yloxy)carbonyl]methoxy\}phenyl- $\kappa C^{1}$ )chlorido(4-methylpyridine- $\left.\kappa N\right)$ platinum(II), 


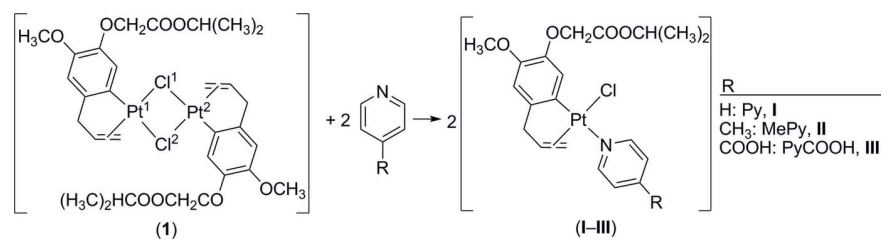

Figure 1

Reaction scheme for the synthesis of mixed ${ }^{i}$ PrEug-pyridine derivative platinum(II) complexes (I), (II) and (III).

$\left[\mathrm{Pt}\left(\mathrm{C}_{15} \mathrm{H}_{19} \mathrm{O}_{4}\right) \mathrm{Cl}\left(\mathrm{C}_{6} \mathrm{H}_{7} \mathrm{~N}\right)\right]$, (II), and $\left(\eta^{2}\right.$-2-allyl-4-methoxy-5$\left\{[(\right.$ propan-2-yloxy)carbonyl $]$ methoxy $\}$ phenyl- $\left.\kappa C^{1}\right)$ chlorido(pyridine-4-carboxylic acid- $\kappa N)$ platinum(II), $\left[\mathrm{Pt}\left(\mathrm{C}_{15} \mathrm{H}_{19} \mathrm{O}_{4}\right)\right.$ $\left.\mathrm{Cl}\left(\mathrm{C}_{6} \mathrm{H}_{5} \mathrm{NO}_{2}\right)\right]$, (III), are reported. Complexes (I), (II), (III) are obtained from the reactions of the dinuclear chelate ring complex $\quad\left[\mathrm{Pt}(\mu-\mathrm{Cl})\left({ }^{i} \mathrm{PrEug}\right)\right]_{2} \quad\left(\mathbf{1}, \quad{ }^{i}\right.$ PrEug: deprotonated isopropyl eugenoxyacetate) with pyridine (Py), 4-methylpyridine (MePy) and pyridine-4-carboxylic acid $(\mathrm{PyCOOH})$, respectively. The synthesis of the three complexes is summarized in Fig. 1.

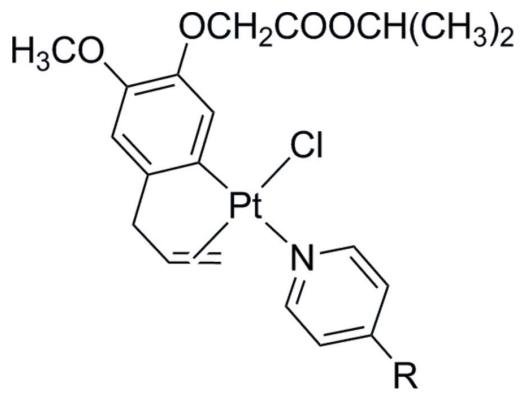

(I) $\mathrm{R}=\mathrm{H}$

(II) $\mathrm{R}=\mathrm{CH}_{3}$

(III) $\mathrm{R}=\mathrm{COOH}$

The Py, MePy and PyCOOH cleave the $\mathrm{Pt}^{1}-\mathrm{Cl}^{2}$ (or $\mathrm{Pt}^{2}-$ $\mathrm{Cl}^{1}$ ) bond in complex 1 to form complexes (I), (II), (III). This is due to the weaker $\mathrm{Pt}^{1}-\mathrm{Cl}^{2}$ or $\mathrm{Pt}^{2}-\mathrm{Cl}^{1}$ bond $(2.4773 \AA)$ as compared to the $\mathrm{Pt}^{1}-\mathrm{Cl}^{1}$ or $\mathrm{Pt}^{2}-\mathrm{Cl}^{2}$ bond $(2.3527 \AA$ ) (Nguyen Thi Thanh et al., 2016) and results in a cis but not trans position of the pyridine ligands with respect to the allyl group of ${ }^{i}$ PrEug. Similar results have been observed when the complexes $[\mathrm{Pt}(\mu-\mathrm{Cl})(\text { arylolefin- } 1 \mathrm{H})]_{2}$ (arylolefin: safrole or eugenol derivatives) analogous to $\mathbf{1}$ react with different amines (Da et al., 2012, 2015; Chi et al., 2018).

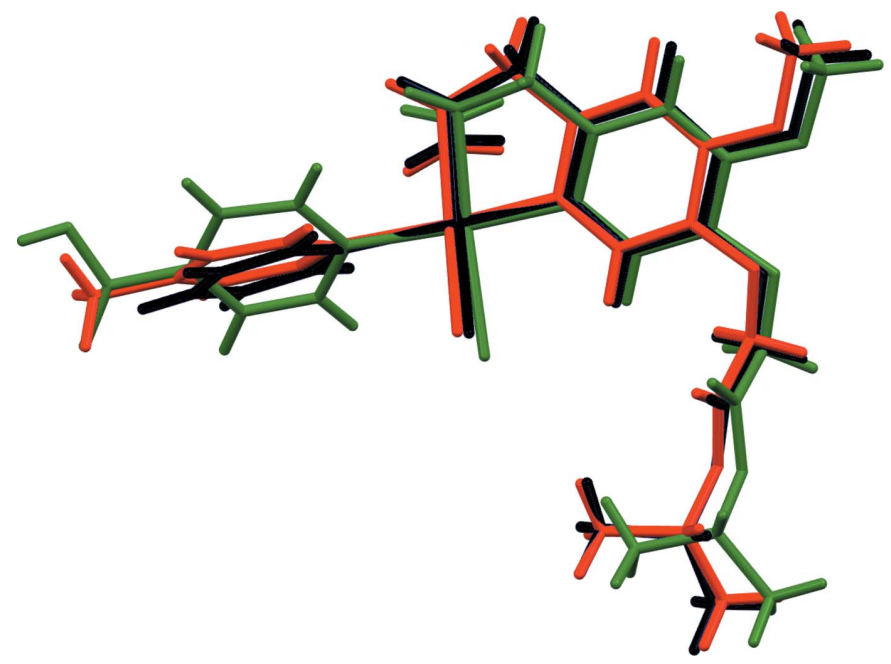

Figure 3

Overlay of the three complexes, showing the different conformation of the pyridine ring for (III). Complex (I) is in black, complex (II) in red and complex (III) in green.

\section{Structural commentary}

Complexes (I) and (II) crystallize isomorphously in the triclinic space group $P \overline{1}$. The central $\mathrm{Pt}^{\mathrm{II}}$ atom displays a distorted square-planar coordination with the $\mathrm{Cl}$ atom, the $\mathrm{N}$ atom of the pyridine or 4-methylpyridine ligand, and completed with a $\mathrm{C}$ atom and $\mathrm{C}=\mathrm{C}$ double bond of the eugenol ligand (Fig. $2 a$ and $2 b$ ). The $\mathrm{C}=\mathrm{C}$ group and $\mathrm{N}$ atom are in a cis position with respect to each other. The dihedral angle between the best planes through the pyridine and phenyl rings is $74.90(15)^{\circ}$ for complex (I) and $75.00(11)^{\circ}$ for complex (II). The dihedral angle between the planes through the allyl atoms $(\mathrm{C} 8, \mathrm{C} 9, \mathrm{C} 10)$ and the pyridine ring is $16.0(2)^{\circ}$ for complex (I) and 20.08 (12) for complex (II). The almost identical conformation is further evidenced by a fit of both structures, excluding $\mathrm{H}$ atoms and the methyl substituent in (II), which gives an r.m.s. deviation of $0.1867 \AA$ (Fig. 3).

Complex (III) also crystallizes in space group $P \overline{1}$, but due to the presence of the carboxylic acid function the crystal structure is no longer isomorphous with (I) and (II) (Fig. 2c). Although the square-planar coordination of the central $\mathrm{Pt}^{\mathrm{II}}$ atom is identical, the dihedral angle of $21.6(2)^{\circ}$ illustrates that

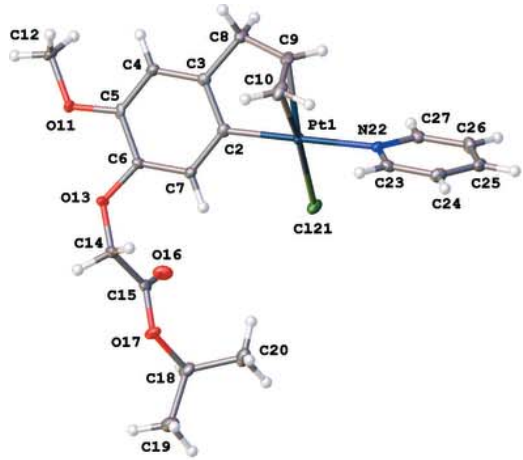

(a)

Figure 2

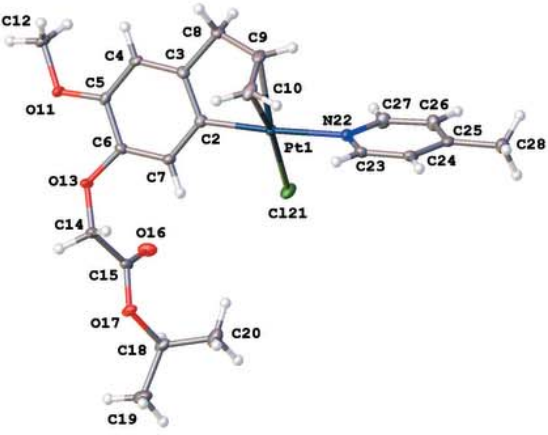

(b)

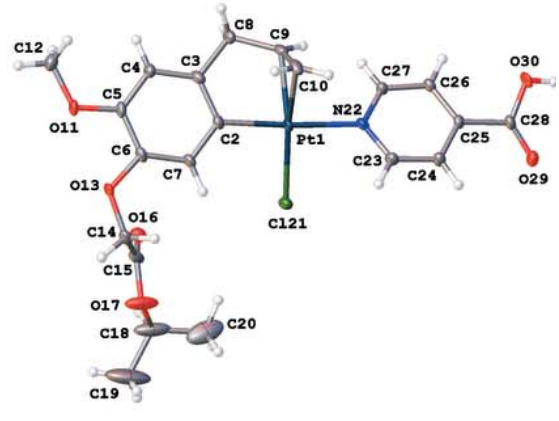

(c)

The molecular structure of complexes (I), (II) and (III) showing the atom-labelling scheme and displacement ellipsoids at the $50 \%$ probability level. 
Table 1

Hydrogen-bond geometry $\left(\AA{ }^{\circ}\right)$ for $(\mathbf{I})$.

$\mathrm{Cg} 5$ is the centroid of the $\mathrm{C} 2-\mathrm{C} 7$ phenyl ring.

\begin{tabular}{lllll}
\hline$D-\mathrm{H} \cdots A$ & $D-\mathrm{H}$ & $\mathrm{H} \cdots A$ & $D \cdots A$ & $D-\mathrm{H} \cdots A$ \\
\hline $\mathrm{C} 12-\mathrm{H} 12 B \cdots \mathrm{O} 16^{\text {i }}$ & 0.98 & 2.42 & $3.354(3)$ & 160 \\
$\mathrm{C} 14-\mathrm{H} 14 A \cdots \mathrm{O} 11^{\text {ii }}$ & 0.99 & 2.31 & $3.266(3)$ & 161 \\
$\mathrm{C} 14-\mathrm{H} 14 A \cdots \mathrm{O} 13^{\text {ii }}$ & 0.99 & 2.56 & $3.330(4)$ & 134 \\
$\mathrm{C} 20-\mathrm{H} 20 B \cdots C g 5^{\text {iii }}$ & 0.98 & 2.93 & $3.586(3)$ & 125 \\
$\mathrm{C} 26-\mathrm{H} 26 \cdots C g 5^{\text {iv }}$ & 0.95 & 2.88 & $3.736(3)$ & 150 \\
\hline
\end{tabular}

Symmetry codes: (i) $x-1, y, z$; (ii) $-x+2,-y,-z+2$; (iii) $x+1, y, z$; (iv) $-x+2,-y+1,-z+1$

the mutual orientation of the eugenol and pyridine parts is different. The plane through the allyl group makes an angle of $40.9(3)^{\circ}$ with the pyridine plane. An overlay of the identical parts in (I) and (III) gives an r.m.s. deviation of $0.5782 \AA$, while $0.5507 \AA$ for (II) and (III) (Fig. 3).

Comparing the bond distances in the coordination sphere of the central $\mathrm{Pt}^{\mathrm{II}}$ atom of the three complexes shows that the largest differences occur for the $\mathrm{Pt}-\mathrm{N}$ distance: 2.139 (2) $\AA$ for (I) within experimental error the same as 2.1418 (18) $\AA$ for (II), and 2.164 (3) $\AA$ for (III).

\section{Supramolecular features}

The crystal packing of complex (I) is characterized by $\mathrm{C}-$ $\mathrm{H} \cdots \mathrm{O}$ hydrogen bonding, $\mathrm{C}-\mathrm{H} \cdots \pi$ and $\pi-\pi$ interactions (Fig. 4, Table 1). The bifurcated hydrogen bond between $\mathrm{C} 14-\mathrm{H} 14 A$ and $\mathrm{O} 11 / \mathrm{O} 13$ gives rise to the formation of inversion dimers. The eugenol parts are further linked into chains running in the $a$-axis direction by $\mathrm{C} 12-\mathrm{H} 12 B \cdots \mathrm{O} 16$ hydrogen-bond interactions. Further dimer formation is obtained through $\pi-\pi$ stacking between the pyridine rings $\left[C g 4 \cdots C g 4^{\mathrm{v}}=3.560\right.$ (2) $\AA$; $C g 4$ is the centroid of ring N22/ C23-C27; symmetry code: (v) $2-x, 2-y, 1-z]$. The phenyl ring $\mathrm{C} 2-\mathrm{C} 7$ participates in two $\mathrm{C}-\mathrm{H} \cdots \pi$ interactions.

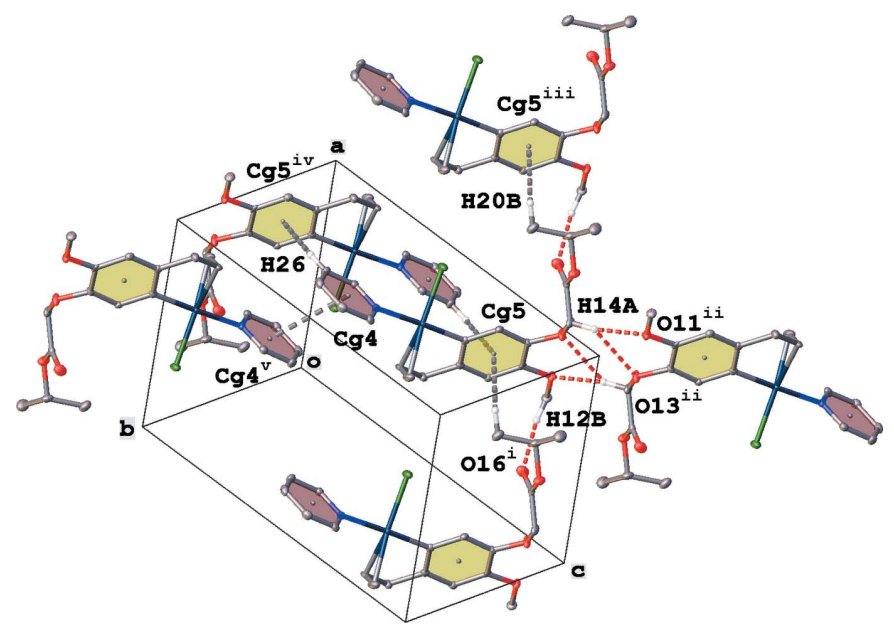

Figure 4

Partial crystal packing of complex (I), showing $\mathrm{C}-\mathrm{H} \cdots \mathrm{O}$ hydrogen bonding (red dashed lines), $\mathrm{C}-\mathrm{H} \cdots \pi$ and $\pi-\pi$ interactions (grey dashed lines). Hydrogen atoms not involved in interactions have been omitted for clarity (see Table 1 for symmetry codes).
Table 2

Hydrogen-bond geometry $\left(\AA,^{\circ}\right)$ for (II).

$\mathrm{Cg} 5$ is the centroid of the $\mathrm{C} 2-\mathrm{C} 7$ phenyl ring.

\begin{tabular}{lllll}
\hline$D-\mathrm{H} \cdots A$ & $D-\mathrm{H}$ & $\mathrm{H} \cdots A$ & $D \cdots A$ & $D-\mathrm{H} \cdots A$ \\
\hline $\mathrm{C} 12-\mathrm{H} 12 B \cdots \mathrm{O} 16^{\mathrm{i}}$ & 0.98 & 2.45 & $3.397(3)$ & 162 \\
$\mathrm{C} 14-\mathrm{H} 14 A \cdots \mathrm{O} 11^{\mathrm{ii}}$ & 0.99 & 2.39 & $3.341(3)$ & 161 \\
$\mathrm{C} 14-\mathrm{H} 14 A \cdots \mathrm{O} 13^{\mathrm{ii}}$ & 0.99 & 2.57 & $3.351(3)$ & 136 \\
$\mathrm{C} 8-\mathrm{H} 8 B \cdots \mathrm{Cl} 21^{\mathrm{i}}$ & 0.99 & 2.76 & $3.713(3)$ & 162 \\
$\mathrm{C} 20-\mathrm{H} 20 B \cdots C g 5^{\mathrm{iii}}$ & 0.98 & 2.87 & $3.562(3)$ & 128 \\
$\mathrm{C} 26-\mathrm{H} 26 \cdots C g 5^{\mathrm{iv}}$ & 0.95 & 2.93 & $3.873(3)$ & 171 \\
$\mathrm{C} 28-\mathrm{H} 28 B \cdots C g 4^{\mathrm{v}}$ & 0.98 & 2.87 & $3.425(3)$ & 117 \\
\hline
\end{tabular}

Symmetry codes: (i) $x-1, y, z$; (ii) $-x+2,-y,-z+2$; (iii) $x+1, y, z$; (iv) $-x+2,-y+1,-z+1 ;(\mathrm{v})-x+2,-y+2,-z+1$.

Table 3

Hydrogen-bond geometry $\left(\AA{ }^{\circ}\right)$ for (III).

\begin{tabular}{|c|c|c|c|c|}
\hline$D-\mathrm{H} \cdots A$ & $D-\mathrm{H}$ & $\mathrm{H} \cdots A$ & $D \cdots A$ & $D-\mathrm{H} \cdots A$ \\
\hline $\mathrm{O} 30-\mathrm{H} 30 \cdots \mathrm{O} 13^{\mathrm{i}}$ & 0.84 & 2.10 & $2.932(4)$ & 170 \\
\hline $\mathrm{C} 10-\mathrm{H} 10 A \cdots \mathrm{O} 16^{\mathrm{ii}}$ & 0.95 & 2.41 & $3.317(5)$ & 159 \\
\hline $\mathrm{C} 12-\mathrm{H} 12 A \cdots \mathrm{O} 16^{\mathrm{iii}}$ & 0.98 & 2.51 & $3.415(5)$ & 154 \\
\hline $\mathrm{C} 14-\mathrm{H} 14 A \cdots \mathrm{O} 29^{\text {iv }}$ & 0.99 & 2.46 & $3.268(5)$ & 139 \\
\hline $\mathrm{C} 14-\mathrm{H} 14 B \cdots \mathrm{O} 29^{\mathrm{v}}$ & 0.99 & 2.46 & $3.178(5)$ & 129 \\
\hline $\mathrm{C} 26-\mathrm{H} 26 \cdots \mathrm{O} 16^{\mathrm{vi}}$ & 0.95 & 2.43 & $3.336(5)$ & 159 \\
\hline
\end{tabular}

Symmetry codes: (i) $x-1, y, z-1$; (ii) $x-1, y, z$; (iii) $-x+1,-y+1,-z+1$; (iv) $x+1, y, z+1 ;(\mathrm{v})-x,-y+2,-z ;$ (vi) $-x,-y+1,-z$.

Complex (II) displays a very similar crystal packing (Fig. 5, Table 2). But, due to the presence of a 4-methylpyridine ring in (II), the $\pi-\pi$ stacking is absent $\left[C g 4 \cdots C g 4^{\mathrm{v}}=4.312\right.$ (1) $\AA$, slippage $2.703 \AA$; $C g 4$ is the centroid of ring N22/C23-C27; symmetry code: (v) $2-x, 2-y, 1-z]$ and is in fact replaced by two $\mathrm{C}-\mathrm{H} \cdots \pi$ interactions between the methyl group and the pyridine ring. This slippage of the pyridine ring also results in an additional $\mathrm{C} 8-\mathrm{H} 8 \mathrm{~B} \cdots \mathrm{Cl} 21$ interaction between the allyl $\mathrm{CH}_{2}$ group and a neighboring $\mathrm{Cl}$ atom.

The carboxylic acid function present in complex (III) is involved in head-to-tail fashion $\mathrm{O}-\mathrm{H} \cdots \mathrm{O}$ interactions resulting in the formation of chains running in the [101]

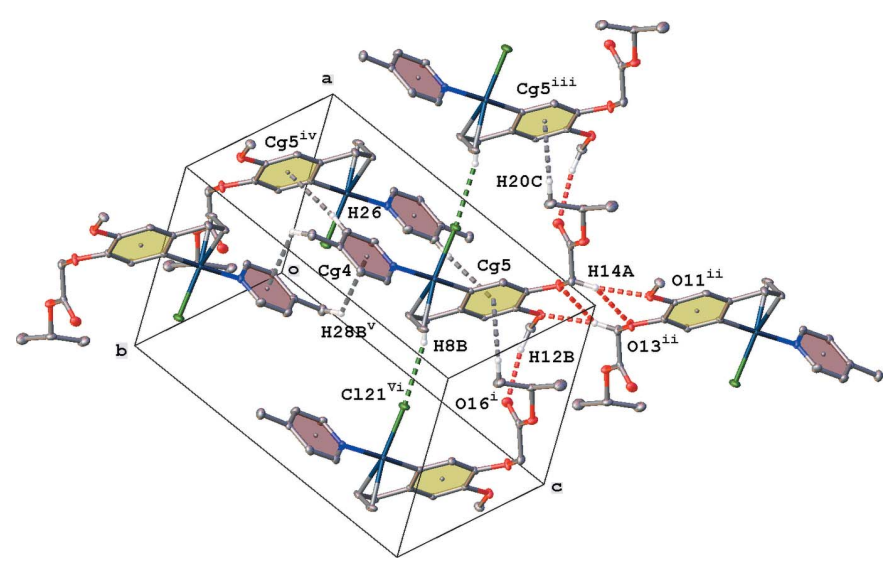

Figure 5

Partial crystal packing of complex (II), showing $\mathrm{C}-\mathrm{H} \cdots \mathrm{O}$ hydrogen bonding (red dashed lines), $\mathrm{C}-\mathrm{H} \cdots \mathrm{Cl}$ (green dashed lines), $\mathrm{C}-\mathrm{H} \cdots \pi$ and $\pi-\pi$ interactions (grey dashed lines). Hydrogen atoms not involved in interactions have been omitted for clarity (see Table 2 for symmetry codes). 
Table 4

Pt bond distances $(\AA)$ for $\mathrm{Pt}$ complexes with the $\mathrm{Pt}$ atom coordinated to a $\mathrm{Cl}$ atom, $\mathrm{N}$ atom and allylaryl ligand found in the Cambridge Structural Database.

$\mathrm{C}_{\text {aryl }}$ is the aryl $\mathrm{C}$ atom and $\mathrm{Cg}$ the centroid of the $\mathrm{C}=\mathrm{C}$ group of the coordinating allylaryl ligand.

\begin{tabular}{llllll}
\hline CSD refcode & $\mathrm{Pt}-\mathrm{Cl}$ & $\mathrm{Pt}-\mathrm{N}$ & $\mathrm{Pt}-\mathrm{C}_{\text {aryl }}$ & $\mathrm{Pt}-C g$ & Reference \\
\hline EWAVOP & 2.323 & 2.107 & 1.995 & 2.011 & Nguyen Thi Thanh et al. (2016) \\
GOYJEL & 2.324 & 2.177 & 2.001 & 2.011 & Da et al. $(2015)$ \\
OFUREN & 2.319 & 2.160 & 2.109 & 2.057 & Da et al. (2008) \\
OFUREN & 2.340 & 2.187 & 1.843 & 1.995 & Da et al. $(2008)$ \\
SOMNUF & 2.329 & 2.188 & 2.015 & 2.009 & Mangwala Kimpende et al. (2014) \\
TALTIM & 2.321 & 2.143 & 2.002 & 2.009 & Le Thi Hong et al. (2017) \\
VEZHOA & 2.332 & 2.140 & 2.006 & 2.007 & Chi et al. (2018) \\
VEZJIW & 2.314 & 2.142 & 1.991 & 2.017 & Chi et al. (2018) \\
VEZJIW & 2.318 & 2.138 & 2.999 & 2.015 & Chi et al. $(2018)$ \\
VEZJOC & 2.317 & 2.199 & & & Chi et al. (2018)
\end{tabular}

direction (Fig. 6, Table 3). Parallel chains interact through $\pi-\pi$ interactions $\left[C g 4 \cdots C g 5^{\mathrm{vi}}=3.947(2) \AA ; C g 4\right.$ and $C g 5$ are the centroids of rings $\mathrm{N} 22 / \mathrm{C} 23-\mathrm{C} 27$ and $\mathrm{C} 2-\mathrm{C} 7$, respectively; symmetry code: (vi) $-x, 1-y,-z]$ and $\mathrm{C}-\mathrm{H} \cdots \mathrm{O}$ hydrogenbonding interactions (Fig. 6, Table 3).

No voids are observed in the crystal packing of complexes (I) and (II), but for complex (III) a small void of $37 \AA^{3}$ is present around $\left(\frac{1}{2}, 0,0\right)$.

\section{Database survey}

A search of the Cambridge Structural Database (CSD, Version 5.41, update of November 2019; Groom et al., 2016) for $\mathrm{Pt}$ complexes with the $\mathrm{Pt}$ atom coordinated to a $\mathrm{Cl}$ atom, $\mathrm{N}$ atom and allylaryl ligand (similar to the title complexes) gave eight hits. The $\mathrm{C}=\mathrm{C}$ group and $\mathrm{N}$ atom are always in a cis position with respect to each other. All complexes also possess a distorted square-planar coordination for the Pt atom with a deviation of the $\mathrm{Pt}$ atom from the best plane through the coordinating $\mathrm{Cl}, \mathrm{N}, \mathrm{C}_{\text {aryl }}$ and centroid $(C g)$ of the $\mathrm{C}=\mathrm{C}$ group between $0.018 \AA$ [chloro-(4,5-dimethoxy-2-prop-2-en-1yl)phenyl-(2-methylaniline)platinum(II), refcode GOYJEL; $\mathrm{Da}$ et al., 2015] and $0.048 \AA$ [( $\eta^{2}-5$-hydroxy-4-methoxy2-(prop-2-en-1-yl)phenyl)-chloro-(4-methylpyridine)platinum(II), CSD refcode VEZJIW; Chi et al., 2018]. Table 4 gives an overview of the four Pt bond distances for each compound. The average $\mathrm{Pt}-\mathrm{Cl}, \mathrm{Pt}-\mathrm{N}, \mathrm{Pt}-\mathrm{C}_{\text {aryl }}$ and $\mathrm{Pt}-C g$ distances are

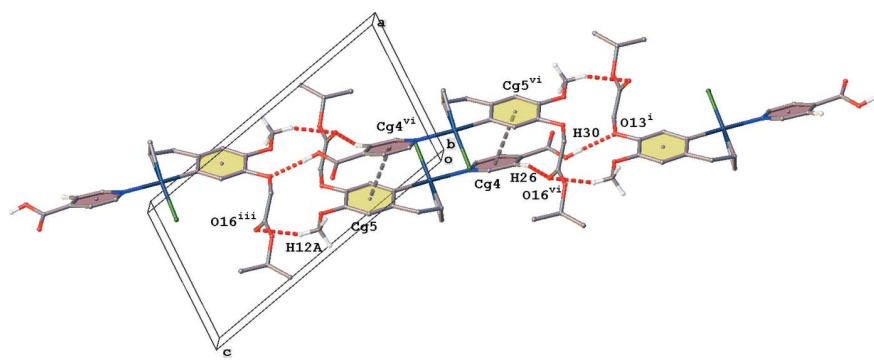

Figure 6

Partial crystal packing of complex (III), showing the chain formation in the [101] direction. $\mathrm{O}-\mathrm{H} \cdots \mathrm{O}$ and $\mathrm{C}-\mathrm{H} \cdots \mathrm{O}$ hydrogen bonding are shown as red dashed lines, $\pi-\pi$ interactions as grey dashed lines. Hydrogen atoms not involved in interactions have been omitted for clarity (see Table 3 for symmetry codes).
2.324 (8), 2.158 (29), 1.996 (64) and 2.014 (16) ^̊, respectively. The largest spread is observed for the $\mathrm{Pt}-\mathrm{C}_{\text {aryl }}$ bond (1.843 to $2.109 \AA$ in the two molecules present in the asymmetric unit of chloro- $\left(\eta^{2}-6\right.$-ethenyl-1,3-benzodioxole-5-yl)piperidine-

platinum(II) (CSD refcode OFUREN; Da et al., 2008). The averages correspond to the observed distances for complexes (I)-(III). It is worthwhile to note that upon binding to Pt, the $\mathrm{C}=\mathrm{C}$ bond distance $[1.29$ (4) $\AA$ for allylaryl fragments in the $\mathrm{CSD}$ ] increased significantly. The average $\mathrm{C}=\mathrm{C}$ bond distance for the complexes in Table 4 is 1.39 (3) $\AA$, comparable to the $\mathrm{C}=\mathrm{C}$ bond distances in the title complexes [1.389(4), 1.401 (3) and 1.376 (6) $\AA$ for (I)-(III), respectively].

\section{In vitro cytotoxicity of complexes (I) and (II)}

The in vitro cytotoxicity of complexes (I) and (II) was tested according to the method described in Skehan et al. (1990) and Likhitwitayawuid et al. (1993) on two human cancer cell lines of HepG2 (hepatocellular carcinoma) and KB (human epidermal carcinoma). The $\mathrm{IC}_{50}$ values for the HepG2 and $\mathrm{KB}$ cell lines calculated based on OD values taken on an Elisa instrument at $515-540 \mathrm{~nm}$ are $150.9,122.3 \mu M$ for (I) and $138.9,93.2 \mu M$ for (II), respectively. This result shows that the presence of the extra methyl group on the pyridine ring in the para position in (II) does not have a notable effect on its anticancer activities as compared to those of (I). However, a comparison of complexes that differ solely in the olefin ligand reveals a significant influence. Specifically, complex (I) exhibits much better cytotoxicity against HepG2 and KB cell lines than $[\mathrm{PtCl}($ eugenol-1H)(Py)] $(>270.7,211.8 \mu M$, respectively; Chi et al., 2018) but worse than $[\mathrm{Pt}($ methyleugenol-1H)(Py)] (7.07 $\mu M$ for KB cell line; Da et al., 2015).

\section{Synthesis and crystallization}

The synthetic protocol for the three complexes is shown in Fig. 1. The starting complex $\left[\mathrm{Pt}(\mu-\mathrm{Cl})\left({ }^{i} \mathrm{PrEug}\right)\right]_{2}$ (1) was synthesized according to the synthetic protocol of Thong \& Chi (2014).

[PtCl( ${ }^{\boldsymbol{i}}$ PrEug)(pyridine)] (I). A solution of pyridine $(80 \mu \mathrm{L}$, $1.0 \mathrm{mmol}$ ) in $10 \mathrm{~mL}$ ethanol was slowly added with stirring to a suspension of $\left[\mathrm{Pt}(\mu-\mathrm{Cl})\left({ }^{i} \mathrm{PrEug}\right)\right]_{2} \quad(494 \mathrm{mg}, 0.5 \mathrm{mmol})$ in 
Table 5

Experimental details.

(I) (II)

Crystal data

Chemical formula

$M_{\mathrm{r}}$

Crystal system, space group

Temperature (K)

$a, b, c(\AA)$

$\alpha, \beta, \gamma\left(^{\circ}\right)$

$V\left(\AA^{3}\right)$

$Z$

Radiation type

$\mu\left(\mathrm{mm}^{-1}\right)$

Crystal size (mm)

Data collection

Diffractometer

Absorption correction

$T_{\min }, T_{\max }$

No. of measured, independent and observed $[I>2 \sigma(I)]$ reflections

$R_{\text {int }}$

$(\sin \theta / \lambda)_{\max }\left(\AA^{-1}\right)$

$\left[\mathrm{Pt}\left(\mathrm{C}_{15} \mathrm{H}_{19} \mathrm{O}_{4}\right) \mathrm{Cl}\left(\mathrm{C}_{5} \mathrm{H}_{5} \mathrm{~N}\right)\right]$

572.94

Triclinic, $P \overline{1}$

100

8.3146 (3), 8.6714 (4), 14.5827 (6)

90.534 (4), 104.376 (4), 101.135 (3)

$997.49(7)$

2

Mo $K \alpha$

7.19

$0.25 \times 0.2 \times 0.15$

Rigaku Oxford Diffraction SuperNova, Single source at offset/far, Eos

Multi-scan CrysAlis PRO (Rigaku OD, 2018)

$0.717,1.000$

$17455,4084,3881$

0.040

0.625

Refinement

$R\left[F^{2}>2 \sigma\left(F^{2}\right)\right], w R\left(F^{2}\right), S$

No. of reflections

No. of parameters

No. of restraints

$\mathrm{H}$-atom treatment

$\Delta \rho_{\max }, \Delta \rho_{\min }\left(\mathrm{e} \AA^{-3}\right)$

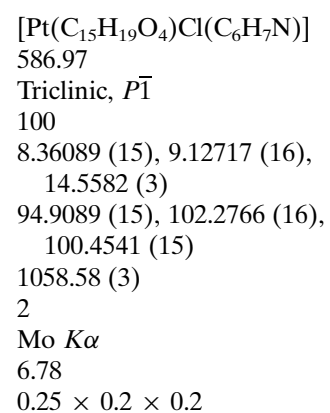

Rigaku Oxford Diffraction SuperNova, Single source at offset/far, Eos

Multi-scan CrysAlis PRO (Rigaku OD, 2018)

$0.671,1.000$

$43513,4327,4252$

\subsection{6}

0.625

$0.013,0.033,1.12$
4327
257
0
H-atom parameters constrained
$0.38,-0.92$

(III)

\author{
$\left[\mathrm{Pt}\left(\mathrm{C}_{15} \mathrm{H}_{19} \mathrm{O}_{4}\right) \mathrm{Cl}\left(\mathrm{C}_{6} \mathrm{H}_{5} \mathrm{NO}_{2}\right)\right]$ \\ 616.95 \\ Triclinic, $P \overline{1}$ \\ 100 \\ 7.8746 (2), 9.7566 (2), 15.0004 (4) \\ $95.782(2), 102.874(2), 93.843$ (2) \\ $1113.02(5)$ \\ 2 \\ Mo $K \alpha$ \\ 6.46 \\ $0.4 \times 0.4 \times 0.35$
}

Rigaku Oxford Diffraction SuperNova, Single source at offset/far, Eos

Multi-scan CrysAlis PRO (Rigaku OD, 2018)

$0.429,1.000$

$22839,4542,4276$

0.077

0.625

$0.027,0.068,1.05$
4542
275
27
H-atom parameters constrained
$1.87,-1.77$

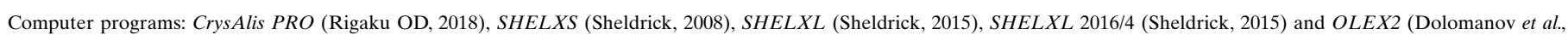
2009).

$10 \mathrm{~mL}$ acetone. The reaction mixture was stirred at ambient temperature (AT) and filtered off after 30 minutes to remove the insoluble part. Subsequently, slow evaporation of the solvent of the obtained solution at AT gave within $10 \mathrm{~h}$ transparent crystals, which were suitable for X-ray diffraction and other analyses. The yield was $515 \mathrm{mg}$ (90\%). \% Pt (found/ calculated): 34.15/34.06. ESI MS ( $\mathrm{m} / \mathrm{z}$, intensity), -MS: 1021 , $100 \%,[2 M-2 \mathrm{Py}+\mathrm{Cl}]^{-}$; +MS: 1067, 100\%, $[2 M-\mathrm{Py}+\mathrm{H}]^{+}$; $988,30 \%,\left[2 M-2 \mathrm{Py}+\mathrm{H}^{+}\right.$. IR $\left(\mathrm{cm}^{-1}, v\right): 3089,2970$ and 2839 $(\mathrm{CH}) ; 1748(\mathrm{C}=\mathrm{O}) ; 1597$ and $1477(\mathrm{C}=\mathrm{C}, \mathrm{C}=\mathrm{N}) .{ }^{1} \mathrm{H}$ NMR $\left(500 \mathrm{MHz}\right.$, acetone- $\left.d_{6}\right): 8.79(\mathrm{ov}, 2 \mathrm{H}, \mathrm{Ar}-\mathrm{H}), 8.04(m, 1 \mathrm{H}$, $\mathrm{Ar}-\mathrm{H}), 7.65(\mathrm{ov}, 2 \mathrm{H}, \mathrm{Ar}-\mathrm{H}), 7.04\left(s,{ }^{3} \mathrm{~J}_{\mathrm{PtH}}=40,1 \mathrm{H}, \mathrm{Ar}-\mathrm{H}\right)$, $6.66(\mathrm{~s}, 1 \mathrm{H}, \mathrm{Ar}-\mathrm{H}), 5.07(\mathrm{~m}, 1 \mathrm{H}, \mathrm{O}-\mathrm{CH}), 4.83\left(\mathrm{~m},{ }^{2} \mathrm{~J}_{\mathrm{PtH}}=\right.$ $\left.70 \mathrm{~Hz}, 1 \mathrm{H}, \mathrm{CH}=\mathrm{CH}_{2}\right), 4.54\left(s, 2 \mathrm{H}, \mathrm{OCH}_{2}\right), 3.81\left[d,{ }^{3} J(\mathrm{H}, \mathrm{H})=\right.$ $\left.13.0 \mathrm{~Hz}, 1 \mathrm{H}, \mathrm{CH}=\mathrm{CH}_{2}\right], 3.78-3.74\left(\right.$ ov $, 2 \mathrm{H}, \mathrm{CH}=\mathrm{CH}_{2}, \mathrm{CH}_{2}-$ $\mathrm{CH}), 3.73\left(s, 3 \mathrm{H}, \mathrm{OCH}_{3}\right), 2.66\left(d,{ }^{2} J(\mathrm{H}, \mathrm{H})=16.5 \mathrm{~Hz},{ }^{3} J_{\mathrm{PtH}}=\right.$ $\left.110 \mathrm{~Hz}, 1 \mathrm{H}, \mathrm{CH}_{2}-\mathrm{CH}\right), 1.27\left[d,{ }^{3} \mathrm{~J}(\mathrm{H}, \mathrm{H})=6.5 \mathrm{~Hz}, 6 \mathrm{H}, \mathrm{CH}-\right.$ $\left.\left(\mathrm{CH}_{3}\right)_{2}\right]$.

[PtCl( ${ }^{i}$ PrEug)(4-methylpyridine)] (II). This complex was prepared starting from $\left[\mathrm{Pt}(\mu-\mathrm{Cl})\left({ }^{i} \mathrm{PrEug}\right)\right]_{2} \quad(494 \mathrm{mg}$, $0.5 \mathrm{mmol})$ and 4-methylpyridine $(100 \mu \mathrm{L}, 1.0 \mathrm{mmol})$ according to the procedure for the synthesis of I. The yield was $539 \mathrm{mg}$ $(92 \%)$, transparent crystals were suitable for X-ray diffraction and other analyses. \%Pt (found/calculated): 32.34/32.25. ESI
MS ( $m / z$, intensity), -MS: 1021, 100\%, [2M - 2MePy $+\mathrm{Cl}]^{-}$; +MS: 1079, 70\%, [2M-MePy + H] $]^{+} ; 986,25 \%,[2 M-2 \mathrm{MePy}+$ $\mathrm{H}]^{+}$;. IR $\left(\mathrm{cm}^{-1}, v\right): 2970,2920$ and $2839(\mathrm{CH}) ; 1748(\mathrm{C}=\mathrm{O})$; 1616 and $1477(\mathrm{C}=\mathrm{C}, \mathrm{C}=\mathrm{N}) .{ }^{1} \mathrm{H}$ NMR $(500 \mathrm{MHz}$, acetone$\left.d_{6}\right): 8.60\left[d,{ }^{3} J(\mathrm{H}, \mathrm{H})=5.5 \mathrm{~Hz}, 2 \mathrm{H}, \mathrm{Ar}-\mathrm{H}\right], 7.46\left[d,{ }^{3} J(\mathrm{H}, \mathrm{H})=\right.$ $5.5 \mathrm{~Hz}, 2 \mathrm{H}, \mathrm{Ar}-\mathrm{H}], 7.04\left(s,{ }^{3} \mathrm{~J}_{\mathrm{PtH}}=40,1 \mathrm{H}, \mathrm{Ar}-\mathrm{H}\right), 6.65(s$, $1 \mathrm{H}, \mathrm{Ar}-\mathrm{H}), 5.07(m, 1 \mathrm{H}, \mathrm{O}-\mathrm{CH}), 4.79\left(m,{ }^{2} J_{\mathrm{PtH}}=70 \mathrm{~Hz}, 1 \mathrm{H}\right.$, $\left.\mathrm{CH}=\mathrm{CH}_{2}\right), 4.54\left(s, 2 \mathrm{H}, \mathrm{OCH}_{2}\right), 3.78\left[d,{ }^{3} \mathrm{~J}(\mathrm{H}, \mathrm{H})=13.0 \mathrm{~Hz}\right.$, $\left.1 \mathrm{H}, \mathrm{CH}=\mathrm{CH}_{2}\right], 3.76-3.74\left(\right.$ ov, $\left.2 \mathrm{H}, \mathrm{CH}=\mathrm{CH}_{2}, \mathrm{CH}_{2}-\mathrm{CH}\right)$, $3.73\left(s, 3 \mathrm{H}, \mathrm{OCH}_{3}\right), 2.64\left[d,{ }^{2} J(\mathrm{H}, \mathrm{H})=16.5 \mathrm{~Hz}, 1 \mathrm{H}, \mathrm{CH}_{2}-\right.$ $\mathrm{CH}], 2.46\left(s, 3 \mathrm{H}, \mathrm{CH}_{3}\right), 1.27\left[d,{ }^{3} \mathrm{~J}(\mathrm{H}, \mathrm{H})=6.5 \mathrm{~Hz}, 6 \mathrm{H}, \mathrm{CH}-\right.$ $\left.\left(\mathrm{CH}_{3}\right)_{2}\right]$.

[PtCl( ${ }^{i}$ PrEug)(pyridine-4-carboxylic acid)] (III). A mixture of pyridine-4-carboxylic acid $(123 \mathrm{mg}, 1.0 \mathrm{mmol})$ and $[\mathrm{Pt}(\mu$ $\left.\mathrm{Cl})\left({ }^{i} \mathrm{PrEug}\right)\right]_{2}(494 \mathrm{mg}, 0.5 \mathrm{mmol})$ in $10 \mathrm{~mL}$ acetone was stirred at AT for $8 \mathrm{~h}$. The resulting precipitate was filtered off and washed consecutively with ethanol $(2 \times 5 \mathrm{~mL})$ and cold chloroform $(2 \times 5 \mathrm{~mL})$, then crystallized in chloroform to give a light-yellow powder. The yield was $493 \mathrm{mg}$ (80\%). Single crystals suitable for X-ray diffraction were obtained by slow evaporation within $8 \mathrm{~h}$ from a concentrated chloroform/ ethanol solution at AT. \%Pt (found/calculated): 31.58/31.63. ESI MS ( $m / z$, intensity), $-M S: 1021,100 \%$, [2M-2PyCOOH + $\mathrm{Cl}]^{-}$; $+\mathrm{MS}: 1110,8 \%$, [2M - $\left.\mathrm{PyCOOH}+\mathrm{H}\right]^{+} ; 989,10 \%,[2 M-$ 
$2 \mathrm{PyCOOH}+\mathrm{H}]^{+}$. IR $\left(\mathrm{cm}^{-1}, v\right): 3267(\mathrm{OH}), 3093,2974$ and $2839(\mathrm{CH}) ; 1728(\mathrm{C}=\mathrm{O}) ; 1586$ and $1477(\mathrm{C}=\mathrm{C}, \mathrm{C}=\mathrm{N}) \cdot{ }^{1} \mathrm{H}$ NMR (500 MHz, dimethyl sulfoxide- $\left.d_{6}\right)$ : $13.80(b r, 1 \mathrm{H}, \mathrm{OH})$, $8.79\left[d,{ }^{3} J(\mathrm{H}, \mathrm{H})=4.5 \mathrm{~Hz}, 2 \mathrm{H}, \mathrm{Ar}-\mathrm{H}\right], 7.83\left[d,{ }^{3} J(\mathrm{H}, \mathrm{H})=\right.$ $4.5 \mathrm{~Hz}, 2 \mathrm{H}, \mathrm{Ar}-\mathrm{H}], 6.75-6.74(\mathrm{ov}, 2 \mathrm{H}, \mathrm{Ar}-\mathrm{H}), 5.08(m, 1 \mathrm{H}$, $\left.\mathrm{CH}=\mathrm{CH}_{2}\right), 4.97(m, 1 \mathrm{H}, \mathrm{O}-\mathrm{CH}), 4.58 / 4.51\left[d,{ }^{2} \mathrm{~J}(\mathrm{H}, \mathrm{H})=\right.$ $\left.16.5 \mathrm{~Hz}, 2 \mathrm{H}, \mathrm{OCH}_{2}\right], 4.33\left[d,{ }^{3} J(\mathrm{H}, \mathrm{H})=6.0 \mathrm{~Hz}, 1 \mathrm{H}\right.$, $\left.\mathrm{CH}=\mathrm{CH}_{2}\right], 3.93\left[d,{ }^{3} \mathrm{~J}(\mathrm{H}, \mathrm{H})=13.5 \mathrm{~Hz}, 1 \mathrm{H}, \mathrm{CH}=\mathrm{CH}_{2}\right], 3.79-$ $3.70\left(\right.$ ov $\left., 4 \mathrm{H}, \mathrm{CH}_{2}-\mathrm{CH}, \mathrm{OCH}_{3}\right), 2.77\left[d,{ }^{2} \mathrm{~J}(\mathrm{H}, \mathrm{H})=17.0 \mathrm{~Hz}\right.$, $\left.1 \mathrm{H}, \mathrm{CH}_{2}-\mathrm{CH}\right], 1.23\left[d,{ }^{3} \mathrm{~J}(\mathrm{H}, \mathrm{H})=6.0 \mathrm{~Hz}, 6 \mathrm{H}, \mathrm{CH}-\left(\mathrm{CH}_{3}\right)_{2}\right]$.

\section{Refinement}

Crystal data, data collection and structure refinement details are summarized in Table 5.

The $\mathrm{H}$ atoms were placed in idealized positions and included as riding contributions with $U_{\text {iso }}(\mathrm{H})$ values of $1.2 U_{\text {eq }}$ or $1.5 U_{\text {eq }}$ of the parent atoms, with $\mathrm{C}-\mathrm{H}$ distances of 0.95 (aromatic), $1.00(\mathrm{CH}), 0.99\left(\mathrm{CH}_{2}\right)$ and $0.98 \AA\left(\mathrm{CH}_{3}\right)$. The carboxylic acid $\mathrm{H}$ atom in (III) was refined as rotating group with a $\mathrm{O}-\mathrm{H}$ distance of $0.84 \AA$. The displacement parameters of the bonded atoms in the carboxylic acid and isopropyl groups in (III) were restrained to be similar along the bond.

\section{Funding information}

LVM thanks the Hercules Foundation for supporting the purchase of the diffractometer through project AKUL/09/ 0035 .

\section{References}

Bigioni, M., Ganis, P., Panunzi, A., Ruffo, F., Salvatore, C. \& Vito, A. (2000). Eur. J. Inorg. Chem. pp. 1717-1721.
Chi, N. T. T., Da, T. T., Ha, N. V. \& Dinh, N. H. (2017). J. Coord. Chem. 70, 1008-1019.

Chi, N. T. T., Da, T. T., Robeyns, K., Van Meervelt, L., Mai, T. T. C., Dat, N. D. \& Dinh, N. H. (2018). Polyhedron, 151, 330-337.

Cucciolito, M. E., D'Amora, A., De Feo, G., Ferraro, G., Giorgio, A., Petruk, G., Monti, D. M., Merlino, A. \& Ruffo, F. (2018). Inorg. Chem. 57, 3133-3143.

Da, T. T., Chi, N. T. T., Van Meervelt, L., Kimpende, P. M. \& Dinh, N. H. (2015). Polyhedron, 85, 104-109.

Da, T. T., Chien, L. X., Chi, N. T. T., Hai, L. T. H. \& Dinh, N. H. (2012). J. Coord. Chem. 65, 131-142.

Da, T. T., Kim, Y. M., Chi, N. T. T., Chien, L. X., Minh, N. V. \& Dinh, N. H. (2008). Organometallics, 27, 3611-3613.

Dodoff, N. I., Lalia-Kantouri, M., Gdaniec, M., Czapik, A., Vassilev, N. G., Markova, L. S. \& Apostolova, M. D. (2012). J. Coord. Chem. 65, 688-704.

Dolomanov, O. V., Bourhis, L. J., Gildea, R. J., Howard, J. A. K. \& Puschmann, H. (2009). J. Appl. Cryst. 42, 339-341.

Groom, C. R., Bruno, I. J., Lightfoot, M. P. \& Ward, S. C. (2016). Acta Cryst. B72, 171-179.

Johnstone, T. C., Suntharalingam, K. \& Lippard, S. J. (2016). Chem. Rev. 116, 3436-3486.

Le Thi Hong, H., Nguyen Thu, T., Nguyen, H. \& Van Meervelt, L. (2017). Acta Cryst. E73, 573-578.

Likhitwitayawuid, K., Angerhofer, C. K., Cordell, G. A., Pezzuto, J. M. \& Ruangrungsi, N. (1993). J. Nat. Prod. 56, 30-38.

Mangwala Kimpende, P., Thi Da, T., Nguyen Huu, D. \& Van Meervelt, L. (2014). Acta Cryst. E70, 435-437.

Nguyen Thi Thanh, C., Pham Van, T., Le Thi Hong, H. \& Van Meervelt, L. (2016). Acta Cryst. C72, 758-764.

Rigaku OD (2018). CrysAlis PRO. Rigaku Oxford Diffraction, Yarnton, England.

Sheldrick, G. M. (2008). Acta Cryst. A64, 112-122.

Sheldrick, G. M. (2015). Acta Cryst. C71, 3-8.

Skehan, P., Storeng, R., Scudiero, D., Monks, A., McMahon, J., Vistica, D., Warren, J. T., Bokesch, H., Kenney, S. \& Boyd, M. R. (1990). J. Natl Cancer Inst. 82, 1107-1112.

Thong, P. V. \& Chi, N. T. T. (2014). J. Chem (Vietnam) 52, 381386. 


\section{supporting information}

Acta Cryst. (2020). E76, 1012-1017 [https://doi.org/10.1107/S2056989020006957]

\section{Crystal structures of three platinacyclic complexes bearing isopropyl}

\section{eugenoxyacetate and pyridine derivatives}

\section{Nguyen Thi Thanh Chi, Pham Van Thong and Luc Van Meervelt}

\section{Computing details}

For all structures, data collection: CrysAlis PRO (Rigaku OD, 2018); cell refinement: CrysAlis PRO (Rigaku OD, 2018); data reduction: CrysAlis PRO (Rigaku OD, 2018); program(s) used to solve structure: SHELXS (Sheldrick, 2008). Program(s) used to refine structure: SHELXL (Sheldrick, 2015) for (I), (II); SHELXL 2016/4 (Sheldrick, 2015) for (III). For all structures, molecular graphics: OLEX2 (Dolomanov et al., 2009); software used to prepare material for publication: OLEX2 (Dolomanov et al., 2009).

( $\eta^{2}$-2-Allyl-4-methoxy-5-\{[(propan-2-yloxy)carbonyl] methoxy\}phenyl- $\left.\kappa C^{1}\right)$ chlorido(pyridine- $\kappa$ N) platinum(II) (I)

Crystal data

$\left[\mathrm{Pt}\left(\mathrm{C}_{15} \mathrm{H}_{19} \mathrm{O}_{4}\right) \mathrm{Cl}\left(\mathrm{C}_{5} \mathrm{H}_{5} \mathrm{~N}\right)\right]$

$M_{r}=572.94$

Triclinic, $P \overline{1}$

$a=8.3146(3) \AA$

$b=8.6714(4) \AA$

$c=14.5827(6) \AA$

$\alpha=90.534(4)^{\circ}$

$\beta=104.376(4)^{\circ}$

$\gamma=101.135(3)^{\circ}$

$V=997.49(7) \AA^{3}$

\section{Data collection}

Rigaku Oxford Diffraction SuperNova, Single source at offset/far, Eos diffractometer

Radiation source: SuperNova (Mo) X-ray Source

Mirror monochromator

Detector resolution: 15.9631 pixels $\mathrm{mm}^{-1}$

$\omega$ scans

Absorption correction: multi-scan

CrysAlisPro (Rigaku OD, 2018)

Refinement

Refinement on $F^{2}$

Least-squares matrix: full

$R\left[F^{2}>2 \sigma\left(F^{2}\right)\right]=0.018$

$w R\left(F^{2}\right)=0.040$

$S=1.05$

4084 reflections
$Z=2$

$F(000)=556$

$D_{\mathrm{x}}=1.908 \mathrm{Mg} \mathrm{m}^{-3}$

Mo $K \alpha$ radiation, $\lambda=0.71073 \AA$

Cell parameters from 12348 reflections

$\theta=3.2-29.0^{\circ}$

$\mu=7.19 \mathrm{~mm}^{-1}$

$T=100 \mathrm{~K}$

Needle, clear colourless

$0.25 \times 0.2 \times 0.15 \mathrm{~mm}$

$T_{\min }=0.717, T_{\max }=1.000$

17455 measured reflections

4084 independent reflections

3881 reflections with $I>2 \sigma(I)$

$R_{\text {int }}=0.040$

$\theta_{\text {max }}=26.4^{\circ}, \theta_{\min }=2.7^{\circ}$

$h=-10 \rightarrow 10$

$k=-10 \rightarrow 10$

$l=-18 \rightarrow 18$

247 parameters

0 restraints

Primary atom site location: heavy-atom method

Hydrogen site location: inferred from neighbouring sites

$\mathrm{H}$-atom parameters constrained 
$w=1 /\left[\sigma^{2}\left(F_{\mathrm{o}}^{2}\right)+(0.0133 P)^{2}+0.0785 P\right]$

where $P=\left(F_{\mathrm{o}}^{2}+2 F_{\mathrm{c}}^{2}\right) / 3$

$(\Delta / \sigma)_{\max }=0.001$
$\Delta \rho_{\max }=0.53$ e $\AA^{-3}$
$\Delta \rho_{\min }=-0.68$ e $\AA^{-3}$

Special details

Geometry. All esds (except the esd in the dihedral angle between two 1.s. planes) are estimated using the full covariance matrix. The cell esds are taken into account individually in the estimation of esds in distances, angles and torsion angles; correlations between esds in cell parameters are only used when they are defined by crystal symmetry. An approximate (isotropic) treatment of cell esds is used for estimating esds involving 1.s. planes.

Fractional atomic coordinates and isotropic or equivalent isotropic displacement parameters $\left(\AA^{2}\right)$

\begin{tabular}{|c|c|c|c|c|}
\hline & $x$ & $y$ & $z$ & $U_{\text {iso }} * / U_{\text {eq }}$ \\
\hline Pt1 & 0.99983 (2) & $0.56342(2)$ & $0.66724(2)$ & $0.00936(4)$ \\
\hline $\mathrm{C} 2$ & $0.9123(4)$ & $0.3610(3)$ & $0.7197(2)$ & $0.0114(6)$ \\
\hline $\mathrm{C} 3$ & $0.7406(3)$ & $0.2958(3)$ & $0.6843(2)$ & $0.0112(6)$ \\
\hline $\mathrm{C} 4$ & $0.6695(4)$ & $0.1536(3)$ & $0.7156(2)$ & $0.0118(6)$ \\
\hline H4 & 0.552907 & 0.109068 & 0.689752 & $0.014 *$ \\
\hline $\mathrm{C} 5$ & $0.7671(4)$ & $0.0764(3)$ & $0.7842(2)$ & $0.0109(6)$ \\
\hline C6 & $0.9385(3)$ & $0.1462(3)$ & $0.8242(2)$ & $0.0107(6)$ \\
\hline $\mathrm{C} 7$ & $1.0104(4)$ & $0.2848(3)$ & $0.79078(19)$ & $0.0111(6)$ \\
\hline $\mathrm{H} 7$ & 1.127197 & 0.328942 & 0.816169 & $0.013 *$ \\
\hline $\mathrm{C} 8$ & $0.6398(4)$ & $0.3846(3)$ & $0.6098(2)$ & $0.0159(7)$ \\
\hline H8A & 0.527756 & 0.383967 & 0.622071 & $0.019^{*}$ \\
\hline $\mathrm{H} 8 \mathrm{~B}$ & 0.621058 & 0.332488 & 0.546305 & $0.019^{*}$ \\
\hline C9 & $0.7352(3)$ & $0.5535(3)$ & $0.6122(2)$ & $0.0165(7)$ \\
\hline H9 & 0.755404 & 0.595040 & 0.555064 & $0.020^{*}$ \\
\hline $\mathrm{C} 10$ & $0.7937(4)$ & $0.6486(4)$ & $0.6956(2)$ & $0.0211(7)$ \\
\hline $\mathrm{H} 10 \mathrm{~A}$ & 0.774433 & 0.608533 & 0.753176 & $0.025^{*}$ \\
\hline H10B & 0.853053 & 0.753727 & 0.695070 & $0.025^{*}$ \\
\hline O11 & $0.7105(2)$ & $-0.0634(2)$ & $0.81928(13)$ & $0.0131(4)$ \\
\hline $\mathrm{C} 12$ & $0.5418(3)$ & -0.1455 & $0.7741(2)$ & $0.0149(7)$ \\
\hline $\mathrm{H} 12 \mathrm{~A}$ & 0.530511 & -0.162299 & 0.706052 & $0.022^{*}$ \\
\hline H12B & 0.460286 & -0.082864 & 0.783549 & $0.022 *$ \\
\hline $\mathrm{H} 12 \mathrm{C}$ & 0.519246 & -0.247470 & 0.801796 & $0.022 *$ \\
\hline $\mathrm{O} 13$ & $1.0221(2)$ & $0.0671(2)$ & $0.89596(14)$ & $0.0170(5)$ \\
\hline $\mathrm{C} 14$ & $1.1676(4)$ & $0.1557(4)$ & $0.9612(2)$ & $0.0159(7)$ \\
\hline H14A & 1.180615 & 0.108580 & 1.023559 & $0.019^{*}$ \\
\hline H14B & 1.151072 & 0.264493 & 0.969509 & $0.019^{*}$ \\
\hline $\mathrm{C} 15$ & $1.3272(4)$ & $0.1608(3)$ & $0.9288(2)$ & $0.0127(6)$ \\
\hline O16 & $1.3354(3)$ & $0.0981(2)$ & $0.85702(14)$ & $0.0194(5)$ \\
\hline $\mathrm{O} 17$ & $1.4603(2)$ & $0.2461(2)$ & $0.99284(14)$ & $0.0159(5)$ \\
\hline $\mathrm{C} 18$ & $1.6258(4)$ & $0.2675(4)$ & $0.9695(2)$ & $0.0175(7)$ \\
\hline H18 & 1.635179 & 0.168389 & 0.937456 & $0.021^{*}$ \\
\hline C19 & $1.7574(4)$ & 0.2994 (4) & 1.0648 (2) & $0.0248(8)$ \\
\hline H19A & 1.743163 & 0.392815 & 1.097858 & $0.037^{*}$ \\
\hline H19B & 1.871372 & 0.317564 & 1.054367 & $0.037^{*}$ \\
\hline H19C & 1.742192 & 0.208415 & 1.103366 & $0.037^{*}$ \\
\hline $\mathrm{C} 20$ & $1.6394(4)$ & $0.4002(4)$ & $0.9044(2)$ & $0.0204(7)$ \\
\hline
\end{tabular}




\begin{tabular}{lllll}
$\mathrm{H} 20 \mathrm{~A}$ & 1.549167 & 0.373773 & 0.845589 & $0.031^{*}$ \\
$\mathrm{H} 20 \mathrm{~B}$ & 1.749964 & 0.416659 & 0.889566 & $0.031^{*}$ \\
$\mathrm{H} 20 \mathrm{C}$ & 1.627884 & 0.496738 & 0.935450 & $0.031^{*}$ \\
$\mathrm{C} 21$ & $1.25878(9)$ & $0.49780(8)$ & $0.67051(5)$ & $0.01618(16)$ \\
$\mathrm{N} 22$ & $1.0890(3)$ & $0.7834(3)$ & $0.61344(17)$ & $0.0113(5)$ \\
$\mathrm{C} 23$ & $1.1235(4)$ & $0.9189(3)$ & $0.6672(2)$ & $0.0140(6)$ \\
$\mathrm{H} 23$ & 1.099025 & 0.916392 & 0.727521 & $0.017^{*}$ \\
$\mathrm{C} 24$ & $1.1935(4)$ & $1.0613(3)$ & $0.6375(2)$ & $0.0157(7)$ \\
$\mathrm{H} 24$ & 1.216748 & 1.155279 & 0.676646 & $0.019^{*}$ \\
$\mathrm{C} 25$ & $1.2290(4)$ & $1.0645(4)$ & $0.5497(2)$ & $0.0168(7)$ \\
$\mathrm{H} 25$ & 1.277421 & 1.160967 & 0.527790 & $0.020^{*}$ \\
$\mathrm{C} 26$ & $1.1935(4)$ & $0.9263(4)$ & $0.4941(2)$ & $0.0169(7)$ \\
$\mathrm{H} 26$ & 1.216671 & 0.926321 & 0.433546 & $0.020^{*}$ \\
$\mathrm{C} 27$ & $1.1239(4)$ & $0.7885(3)$ & $0.5282(2)$ & $0.0136(6)$ \\
$\mathrm{H} 27$ & 1.099668 & 0.693346 & 0.490065 & $0.016^{*}$ \\
\hline
\end{tabular}

Atomic displacement parameters $\left(\AA^{2}\right)$

\begin{tabular}{|c|c|c|c|c|c|c|}
\hline & $U^{11}$ & $U^{22}$ & $U^{33}$ & $U^{12}$ & $U^{13}$ & $U^{23}$ \\
\hline Pt1 & $0.00967(6)$ & $0.00807(6)$ & $0.01093(7)$ & $0.00194(4)$ & $0.00356(5)$ & $0.00123(4)$ \\
\hline $\mathrm{C} 2$ & $0.0130(15)$ & $0.0100(14)$ & $0.0118(15)$ & $0.0020(11)$ & $0.0044(12)$ & $-0.0017(12)$ \\
\hline $\mathrm{C} 3$ & $0.0122(14)$ & $0.0114(14)$ & $0.0104(14)$ & $0.0028(11)$ & $0.0035(12)$ & $-0.0017(12)$ \\
\hline $\mathrm{C} 4$ & $0.0105(14)$ & $0.0130(15)$ & $0.0113(15)$ & $0.0007(11)$ & $0.0031(12)$ & $-0.0014(12)$ \\
\hline $\mathrm{C} 5$ & $0.0160(15)$ & $0.0102(14)$ & $0.0092(14)$ & $0.0018(11)$ & $0.0088(12)$ & $0.0000(12)$ \\
\hline C6 & $0.0103(14)$ & $0.0106(14)$ & $0.0105(14)$ & $0.0038(11)$ & $0.0002(12)$ & $0.0004(12)$ \\
\hline $\mathrm{C} 7$ & $0.0092(14)$ & $0.0132(15)$ & $0.0110(15)$ & $0.0014(11)$ & $0.0036(12)$ & $0.0003(12)$ \\
\hline $\mathrm{C} 8$ & $0.0119(15)$ & $0.0150(16)$ & $0.0212(17)$ & $0.0039(12)$ & $0.0041(13)$ & $0.0050(13)$ \\
\hline C9 & $0.0084(14)$ & $0.0181(16)$ & $0.0261(18)$ & $0.0068(12)$ & $0.0070(13)$ & 0.0089 (14) \\
\hline $\mathrm{C} 10$ & $0.0184(17)$ & $0.0163(16)$ & $0.034(2)$ & $0.0053(13)$ & $0.0153(15)$ & $0.0048(15)$ \\
\hline O11 & $0.0121(10)$ & $0.0111(10)$ & $0.0122(10)$ & $-0.0033(8)$ & $0.0003(8)$ & $0.0022(8)$ \\
\hline $\mathrm{C} 12$ & $0.0095(14)$ & $0.0149(15)$ & $0.0175(16)$ & $-0.0030(12)$ & $0.0021(12)$ & $0.0005(13)$ \\
\hline $\mathrm{O} 13$ & $0.0117(11)$ & $0.0160(11)$ & $0.0168(11)$ & $-0.0030(8)$ & $-0.0040(9)$ & $0.0072(9)$ \\
\hline $\mathrm{C} 14$ & $0.0125(15)$ & $0.0213(17)$ & $0.0104(15)$ & $-0.0008(13)$ & $-0.0003(12)$ & $0.0051(13)$ \\
\hline $\mathrm{C} 15$ & $0.0132(15)$ & $0.0096(14)$ & $0.0140(15)$ & $0.0023(11)$ & $0.0012(12)$ & $0.0037(12)$ \\
\hline O16 & $0.0184(12)$ & $0.0210(12)$ & $0.0160(11)$ & $0.0036(9)$ & $-0.0004(9)$ & $-0.0072(10)$ \\
\hline O17 & $0.0106(10)$ & $0.0203(11)$ & $0.0137(11)$ & $-0.0026(8)$ & $0.0019(9)$ & $-0.0044(9)$ \\
\hline $\mathrm{C} 18$ & $0.0124(15)$ & $0.0224(17)$ & $0.0183(16)$ & $0.0009(13)$ & $0.0073(13)$ & $-0.0015(14)$ \\
\hline C19 & $0.0159(17)$ & $0.032(2)$ & $0.0223(18)$ & $-0.0008(14)$ & $0.0018(14)$ & $0.0025(16)$ \\
\hline $\mathrm{C} 20$ & $0.0222(17)$ & $0.0204(17)$ & $0.0188(17)$ & $-0.0016(13)$ & $0.0100(14)$ & $-0.0001(14)$ \\
\hline $\mathrm{Cl} 21$ & $0.0109(3)$ & $0.0124(4)$ & $0.0265(4)$ & 0.0028 (3) & $0.0067(3)$ & $0.0034(3)$ \\
\hline $\mathrm{N} 22$ & $0.0079(12)$ & $0.0111(12)$ & $0.0140(13)$ & $0.0013(9)$ & $0.0013(10)$ & $0.0014(10)$ \\
\hline $\mathrm{C} 23$ & $0.0136(15)$ & $0.0151(15)$ & $0.0126(15)$ & $0.0052(12)$ & $0.0001(12)$ & 0.0017 (13) \\
\hline $\mathrm{C} 24$ & $0.0147(15)$ & $0.0122(15)$ & $0.0189(16)$ & $0.0020(12)$ & $0.0027(13)$ & $-0.0023(13)$ \\
\hline $\mathrm{C} 25$ & $0.0129(15)$ & $0.0138(15)$ & $0.0215(17)$ & $0.0011(12)$ & $0.0011(13)$ & $0.0056(13)$ \\
\hline $\mathrm{C} 26$ & $0.0190(16)$ & $0.0212(17)$ & $0.0131(15)$ & $0.0057(13)$ & $0.0075(13)$ & $0.0044(13)$ \\
\hline $\mathrm{C} 27$ & $0.0121(15)$ & $0.0149(15)$ & $0.0142(15)$ & $0.0046(12)$ & $0.0024(12)$ & $0.0012(13)$ \\
\hline
\end{tabular}


Geometric parameters $\left(\AA,{ }^{\circ}\right)$

\begin{tabular}{|c|c|c|c|}
\hline $\mathrm{Pt} 1-\mathrm{C} 2$ & $2.001(3)$ & $\mathrm{O} 13-\mathrm{C} 14$ & $1.419(3)$ \\
\hline $\mathrm{Pt} 1-\mathrm{C} 9$ & $2.131(3)$ & $\mathrm{C} 14-\mathrm{H} 14 \mathrm{~A}$ & 0.9900 \\
\hline $\mathrm{Pt} 1-\mathrm{C} 10$ & $2.118(3)$ & $\mathrm{C} 14-\mathrm{H} 14 \mathrm{~B}$ & 0.9900 \\
\hline $\mathrm{Pt} 1-\mathrm{C} 121$ & $2.3205(7)$ & $\mathrm{C} 14-\mathrm{C} 15$ & $1.509(4)$ \\
\hline Pt1-N22 & $2.139(2)$ & $\mathrm{C} 15-\mathrm{O} 16$ & $1.198(3)$ \\
\hline $\mathrm{C} 2-\mathrm{C} 3$ & $1.393(4)$ & $\mathrm{C} 15-\mathrm{O} 17$ & $1.342(3)$ \\
\hline $\mathrm{C} 2-\mathrm{C} 7$ & $1.407(4)$ & $\mathrm{O} 17-\mathrm{C} 18$ & $1.476(3)$ \\
\hline $\mathrm{C} 3-\mathrm{C} 4$ & $1.393(4)$ & $\mathrm{C} 18-\mathrm{H} 18$ & 1.0000 \\
\hline $\mathrm{C} 3-\mathrm{C} 8$ & $1.513(4)$ & $\mathrm{C} 18-\mathrm{C} 19$ & $1.525(4)$ \\
\hline $\mathrm{C} 4-\mathrm{H} 4$ & 0.9500 & $\mathrm{C} 18-\mathrm{C} 20$ & $1.505(4)$ \\
\hline $\mathrm{C} 4-\mathrm{C} 5$ & $1.388(4)$ & $\mathrm{C} 19-\mathrm{H} 19 \mathrm{~A}$ & 0.9800 \\
\hline $\mathrm{C} 5-\mathrm{C} 6$ & $1.409(4)$ & C19-H19B & 0.9800 \\
\hline $\mathrm{C} 5-\mathrm{O} 11$ & $1.365(3)$ & $\mathrm{C} 19-\mathrm{H} 19 \mathrm{C}$ & 0.9800 \\
\hline $\mathrm{C} 6-\mathrm{C} 7$ & $1.384(4)$ & $\mathrm{C} 20-\mathrm{H} 20 \mathrm{~A}$ & 0.9800 \\
\hline $\mathrm{C} 6-\mathrm{O} 13$ & $1.373(3)$ & $\mathrm{C} 20-\mathrm{H} 20 \mathrm{~B}$ & 0.9800 \\
\hline $\mathrm{C} 7-\mathrm{H} 7$ & 0.9500 & $\mathrm{C} 20-\mathrm{H} 20 \mathrm{C}$ & 0.9800 \\
\hline $\mathrm{C} 8-\mathrm{H} 8 \mathrm{~A}$ & 0.9900 & $\mathrm{~N} 22-\mathrm{C} 23$ & $1.349(4)$ \\
\hline $\mathrm{C} 8-\mathrm{H} 8 \mathrm{~B}$ & 0.9900 & $\mathrm{~N} 22-\mathrm{C} 27$ & $1.344(4)$ \\
\hline $\mathrm{C} 8-\mathrm{C} 9$ & $1.520(4)$ & $\mathrm{C} 23-\mathrm{H} 23$ & 0.9500 \\
\hline C9-H9 & 0.9500 & $\mathrm{C} 23-\mathrm{C} 24$ & $1.381(4)$ \\
\hline $\mathrm{C} 9-\mathrm{C} 10$ & $1.389(4)$ & $\mathrm{C} 24-\mathrm{H} 24$ & 0.9500 \\
\hline $\mathrm{C} 10-\mathrm{H} 10 \mathrm{~A}$ & 0.9500 & $\mathrm{C} 24-\mathrm{C} 25$ & $1.383(4)$ \\
\hline $\mathrm{C} 10-\mathrm{H} 10 \mathrm{~B}$ & 0.9500 & $\mathrm{C} 25-\mathrm{H} 25$ & 0.9500 \\
\hline $\mathrm{O} 11-\mathrm{C} 12$ & $1.434(3)$ & $\mathrm{C} 25-\mathrm{C} 26$ & $1.380(4)$ \\
\hline $\mathrm{C} 12-\mathrm{H} 12 \mathrm{~A}$ & 0.9800 & $\mathrm{C} 26-\mathrm{H} 26$ & 0.9500 \\
\hline $\mathrm{C} 12-\mathrm{H} 12 \mathrm{~B}$ & 0.9800 & $\mathrm{C} 26-\mathrm{C} 27$ & $1.378(4)$ \\
\hline $\mathrm{C} 12-\mathrm{H} 12 \mathrm{C}$ & 0.9800 & $\mathrm{C} 27-\mathrm{H} 27$ & 0.9500 \\
\hline $\mathrm{C} 2-\mathrm{Pt} 1-\mathrm{C} 9$ & $81.36(12)$ & $\mathrm{H} 12 \mathrm{~A}-\mathrm{C} 12-\mathrm{H} 12 \mathrm{~B}$ & 109.5 \\
\hline $\mathrm{C} 2-\mathrm{Pt} 1-\mathrm{C} 10$ & $87.41(12)$ & $\mathrm{H} 12 \mathrm{~A}-\mathrm{C} 12-\mathrm{H} 12 \mathrm{C}$ & 109.5 \\
\hline $\mathrm{C} 2-\mathrm{Pt} 1-\mathrm{Cl} 21$ & $93.43(9)$ & $\mathrm{H} 12 \mathrm{~B}-\mathrm{C} 12-\mathrm{H} 12 \mathrm{C}$ & 109.5 \\
\hline $\mathrm{C} 2-\mathrm{Pt} 1-\mathrm{N} 22$ & $178.21(10)$ & $\mathrm{C} 6-\mathrm{O} 13-\mathrm{C} 14$ & $117.1(2)$ \\
\hline $\mathrm{C} 9-\mathrm{Pt} 1-\mathrm{Cl} 21$ & $154.99(9)$ & $\mathrm{O} 13-\mathrm{C} 14-\mathrm{H} 14 \mathrm{~A}$ & 109.1 \\
\hline $\mathrm{C} 9-\mathrm{Pt} 1-\mathrm{N} 22$ & $97.67(10)$ & $\mathrm{O} 13-\mathrm{C} 14-\mathrm{H} 14 \mathrm{~B}$ & 109.1 \\
\hline $\mathrm{C} 10-\mathrm{Pt} 1-\mathrm{C} 9$ & $38.16(12)$ & $\mathrm{O} 13-\mathrm{C} 14-\mathrm{C} 15$ & $112.3(2)$ \\
\hline $\mathrm{C} 10-\mathrm{Pt} 1-\mathrm{Cl} 21$ & $166.72(9)$ & $\mathrm{H} 14 \mathrm{~A}-\mathrm{C} 14-\mathrm{H} 14 \mathrm{~B}$ & 107.9 \\
\hline $\mathrm{C} 10-\mathrm{Pt} 1-\mathrm{N} 22$ & $90.90(11)$ & $\mathrm{C} 15-\mathrm{C} 14-\mathrm{H} 14 \mathrm{~A}$ & 109.1 \\
\hline $\mathrm{N} 22-\mathrm{Pt} 1-\mathrm{Cl} 21$ & $88.08(7)$ & $\mathrm{C} 15-\mathrm{C} 14-\mathrm{H} 14 \mathrm{~B}$ & 109.1 \\
\hline $\mathrm{C} 3-\mathrm{C} 2-\mathrm{Pt} 1$ & $116.6(2)$ & $\mathrm{O} 16-\mathrm{C} 15-\mathrm{C} 14$ & $125.5(3)$ \\
\hline $\mathrm{C} 3-\mathrm{C} 2-\mathrm{C} 7$ & $118.8(3)$ & $\mathrm{O} 16-\mathrm{C} 15-\mathrm{O} 17$ & $124.6(3)$ \\
\hline $\mathrm{C} 7-\mathrm{C} 2-\mathrm{Pt} 1$ & $124.6(2)$ & $\mathrm{O} 17-\mathrm{C} 15-\mathrm{C} 14$ & $109.8(2)$ \\
\hline $\mathrm{C} 2-\mathrm{C} 3-\mathrm{C} 8$ & $116.7(3)$ & $\mathrm{C} 15-\mathrm{O} 17-\mathrm{C} 18$ & $116.4(2)$ \\
\hline $\mathrm{C} 4-\mathrm{C} 3-\mathrm{C} 2$ & $120.5(3)$ & $\mathrm{O} 17-\mathrm{C} 18-\mathrm{H} 18$ & 109.6 \\
\hline $\mathrm{C} 4-\mathrm{C} 3-\mathrm{C} 8$ & $122.8(2)$ & $\mathrm{O} 17-\mathrm{C} 18-\mathrm{C} 19$ & $105.1(2)$ \\
\hline $\mathrm{C} 3-\mathrm{C} 4-\mathrm{H} 4$ & 119.6 & $\mathrm{O} 17-\mathrm{C} 18-\mathrm{C} 20$ & $109.0(3)$ \\
\hline $\mathrm{C} 5-\mathrm{C} 4-\mathrm{C} 3$ & $120.7(3)$ & $\mathrm{C} 19-\mathrm{C} 18-\mathrm{H} 18$ & 109.6 \\
\hline
\end{tabular}




\begin{tabular}{|c|c|c|c|}
\hline $\mathrm{C} 5-\mathrm{C} 4-\mathrm{H} 4$ & 119.6 & $\mathrm{C} 20-\mathrm{C} 18-\mathrm{H} 18$ & 109.6 \\
\hline $\mathrm{C} 4-\mathrm{C} 5-\mathrm{C} 6$ & $119.1(3)$ & $\mathrm{C} 20-\mathrm{C} 18-\mathrm{C} 19$ & $113.7(3)$ \\
\hline $\mathrm{O} 11-\mathrm{C} 5-\mathrm{C} 4$ & $125.5(3)$ & $\mathrm{C} 18-\mathrm{C} 19-\mathrm{H} 19 \mathrm{~A}$ & 109.5 \\
\hline $\mathrm{O} 11-\mathrm{C} 5-\mathrm{C} 6$ & $115.5(3)$ & $\mathrm{C} 18-\mathrm{C} 19-\mathrm{H} 19 \mathrm{~B}$ & 109.5 \\
\hline $\mathrm{C} 7-\mathrm{C} 6-\mathrm{C} 5$ & $120.0(3)$ & $\mathrm{C} 18-\mathrm{C} 19-\mathrm{H} 19 \mathrm{C}$ & 109.5 \\
\hline $\mathrm{O} 13-\mathrm{C} 6-\mathrm{C} 5$ & $115.1(2)$ & $\mathrm{H} 19 \mathrm{~A}-\mathrm{C} 19-\mathrm{H} 19 \mathrm{~B}$ & 109.5 \\
\hline $\mathrm{O} 13-\mathrm{C} 6-\mathrm{C} 7$ & $124.9(2)$ & $\mathrm{H} 19 \mathrm{~A}-\mathrm{C} 19-\mathrm{H} 19 \mathrm{C}$ & 109.5 \\
\hline $\mathrm{C} 2-\mathrm{C} 7-\mathrm{H} 7$ & 119.6 & $\mathrm{H} 19 \mathrm{~B}-\mathrm{C} 19-\mathrm{H} 19 \mathrm{C}$ & 109.5 \\
\hline $\mathrm{C} 6-\mathrm{C} 7-\mathrm{C} 2$ & $120.7(3)$ & $\mathrm{C} 18-\mathrm{C} 20-\mathrm{H} 20 \mathrm{~A}$ & 109.5 \\
\hline $\mathrm{C} 6-\mathrm{C} 7-\mathrm{H} 7$ & 119.6 & $\mathrm{C} 18-\mathrm{C} 20-\mathrm{H} 20 \mathrm{~B}$ & 109.5 \\
\hline $\mathrm{C} 3-\mathrm{C} 8-\mathrm{H} 8 \mathrm{~A}$ & 109.7 & $\mathrm{C} 18-\mathrm{C} 20-\mathrm{H} 20 \mathrm{C}$ & 109.5 \\
\hline $\mathrm{C} 3-\mathrm{C} 8-\mathrm{H} 8 \mathrm{~B}$ & 109.7 & $\mathrm{H} 20 \mathrm{~A}-\mathrm{C} 20-\mathrm{H} 20 \mathrm{~B}$ & 109.5 \\
\hline $\mathrm{C} 3-\mathrm{C} 8-\mathrm{C} 9$ & $109.8(2)$ & $\mathrm{H} 20 \mathrm{~A}-\mathrm{C} 20-\mathrm{H} 20 \mathrm{C}$ & 109.5 \\
\hline $\mathrm{H} 8 \mathrm{~A}-\mathrm{C} 8-\mathrm{H} 8 \mathrm{~B}$ & 108.2 & $\mathrm{H} 20 \mathrm{~B}-\mathrm{C} 20-\mathrm{H} 20 \mathrm{C}$ & 109.5 \\
\hline $\mathrm{C} 9-\mathrm{C} 8-\mathrm{H} 8 \mathrm{~A}$ & 109.7 & $\mathrm{C} 23-\mathrm{N} 22-\mathrm{Pt} 1$ & $120.7(2)$ \\
\hline $\mathrm{C} 9-\mathrm{C} 8-\mathrm{H} 8 \mathrm{~B}$ & 109.7 & $\mathrm{C} 27-\mathrm{N} 22-\mathrm{Pt} 1$ & $120.71(19)$ \\
\hline $\mathrm{Pt} 1-\mathrm{C} 9-\mathrm{H} 9$ & 90.2 & $\mathrm{C} 27-\mathrm{N} 22-\mathrm{C} 23$ & $118.4(2)$ \\
\hline $\mathrm{C} 8-\mathrm{C} 9-\mathrm{Pt} 1$ & $109.4(2)$ & $\mathrm{N} 22-\mathrm{C} 23-\mathrm{H} 23$ & 118.9 \\
\hline $\mathrm{C} 8-\mathrm{C} 9-\mathrm{H} 9$ & 119.1 & $\mathrm{~N} 22-\mathrm{C} 23-\mathrm{C} 24$ & $122.1(3)$ \\
\hline $\mathrm{C} 10-\mathrm{C} 9-\mathrm{Pt} 1$ & $70.44(17)$ & $\mathrm{C} 24-\mathrm{C} 23-\mathrm{H} 23$ & 118.9 \\
\hline $\mathrm{C} 10-\mathrm{C} 9-\mathrm{C} 8$ & $121.7(3)$ & $\mathrm{C} 23-\mathrm{C} 24-\mathrm{H} 24$ & 120.6 \\
\hline $\mathrm{C} 10-\mathrm{C} 9-\mathrm{H} 9$ & 119.1 & $\mathrm{C} 23-\mathrm{C} 24-\mathrm{C} 25$ & $118.7(3)$ \\
\hline $\mathrm{Pt} 1-\mathrm{C} 10-\mathrm{H} 10 \mathrm{~A}$ & 108.6 & $\mathrm{C} 25-\mathrm{C} 24-\mathrm{H} 24$ & 120.6 \\
\hline Pt1-C10-H10B & 90.0 & $\mathrm{C} 24-\mathrm{C} 25-\mathrm{H} 25$ & 120.3 \\
\hline $\mathrm{C} 9-\mathrm{C} 10-\mathrm{Pt} 1$ & $71.40(18)$ & $\mathrm{C} 26-\mathrm{C} 25-\mathrm{C} 24$ & $119.4(3)$ \\
\hline $\mathrm{C} 9-\mathrm{C} 10-\mathrm{H} 10 \mathrm{~A}$ & 120.0 & $\mathrm{C} 26-\mathrm{C} 25-\mathrm{H} 25$ & 120.3 \\
\hline C9- $\mathrm{C} 10-\mathrm{H} 10 \mathrm{~B}$ & 120.0 & $\mathrm{C} 25-\mathrm{C} 26-\mathrm{H} 26$ & 120.6 \\
\hline $\mathrm{H} 10 \mathrm{~A}-\mathrm{C} 10-\mathrm{H} 10 \mathrm{~B}$ & 120.0 & $\mathrm{C} 27-\mathrm{C} 26-\mathrm{C} 25$ & $118.8(3)$ \\
\hline $\mathrm{C} 5-\mathrm{O} 11-\mathrm{C} 12$ & $117.1(2)$ & $\mathrm{C} 27-\mathrm{C} 26-\mathrm{H} 26$ & 120.6 \\
\hline $\mathrm{O} 11-\mathrm{C} 12-\mathrm{H} 12 \mathrm{~A}$ & 109.5 & $\mathrm{~N} 22-\mathrm{C} 27-\mathrm{C} 26$ & $122.5(3)$ \\
\hline $\mathrm{O} 11-\mathrm{C} 12-\mathrm{H} 12 \mathrm{~B}$ & 109.5 & $\mathrm{~N} 22-\mathrm{C} 27-\mathrm{H} 27$ & 118.7 \\
\hline $\mathrm{O} 11-\mathrm{C} 12-\mathrm{H} 12 \mathrm{C}$ & 109.5 & $\mathrm{C} 26-\mathrm{C} 27-\mathrm{H} 27$ & 118.7 \\
\hline $\mathrm{Pt} 1-\mathrm{C} 2-\mathrm{C} 3-\mathrm{C} 4$ & $178.9(2)$ & $\mathrm{C} 7-\mathrm{C} 2-\mathrm{C} 3-\mathrm{C} 4$ & $-3.1(4)$ \\
\hline $\mathrm{Pt} 1-\mathrm{C} 2-\mathrm{C} 3-\mathrm{C} 8$ & $0.3(3)$ & $\mathrm{C} 7-\mathrm{C} 2-\mathrm{C} 3-\mathrm{C} 8$ & $178.4(3)$ \\
\hline $\mathrm{Pt} 1-\mathrm{C} 2-\mathrm{C} 7-\mathrm{C} 6$ & $178.8(2)$ & $\mathrm{C} 7-\mathrm{C} 6-\mathrm{O} 13-\mathrm{C} 14$ & $22.9(4)$ \\
\hline $\mathrm{Pt} 1-\mathrm{N} 22-\mathrm{C} 23-\mathrm{C} 24$ & $174.9(2)$ & $\mathrm{C} 8-\mathrm{C} 3-\mathrm{C} 4-\mathrm{C} 5$ & $-179.9(3)$ \\
\hline $\mathrm{Pt} 1-\mathrm{N} 22-\mathrm{C} 27-\mathrm{C} 26$ & $-175.0(2)$ & $\mathrm{C} 8-\mathrm{C} 9-\mathrm{C} 10-\mathrm{Pt} 1$ & $101.2(3)$ \\
\hline $\mathrm{C} 2-\mathrm{C} 3-\mathrm{C} 4-\mathrm{C} 5$ & $1.6(4)$ & $\mathrm{O} 11-\mathrm{C} 5-\mathrm{C} 6-\mathrm{C} 7$ & $177.3(2)$ \\
\hline $\mathrm{C} 2-\mathrm{C} 3-\mathrm{C} 8-\mathrm{C} 9$ & $-18.2(4)$ & $\mathrm{O} 11-\mathrm{C} 5-\mathrm{C} 6-\mathrm{O} 13$ & $-2.8(4)$ \\
\hline $\mathrm{C} 3-\mathrm{C} 2-\mathrm{C} 7-\mathrm{C} 6$ & $0.9(4)$ & $\mathrm{O} 13-\mathrm{C} 6-\mathrm{C} 7-\mathrm{C} 2$ & $-177.2(3)$ \\
\hline $\mathrm{C} 3-\mathrm{C} 4-\mathrm{C} 5-\mathrm{C} 6$ & $2.1(4)$ & $\mathrm{O} 13-\mathrm{C} 14-\mathrm{C} 15-\mathrm{O} 16$ & $-0.4(4)$ \\
\hline $\mathrm{C} 3-\mathrm{C} 4-\mathrm{C} 5-\mathrm{O} 11$ & $-179.6(3)$ & $\mathrm{O} 13-\mathrm{C} 14-\mathrm{C} 15-\mathrm{O} 17$ & $179.9(2)$ \\
\hline $\mathrm{C} 3-\mathrm{C} 8-\mathrm{C} 9-\mathrm{Pt} 1$ & $25.9(3)$ & $\mathrm{C} 14-\mathrm{C} 15-\mathrm{O} 17-\mathrm{C} 18$ & $-177.7(2)$ \\
\hline $\mathrm{C} 3-\mathrm{C} 8-\mathrm{C} 9-\mathrm{C} 10$ & $-52.5(4)$ & $\mathrm{C} 15-\mathrm{O} 17-\mathrm{C} 18-\mathrm{C} 19$ & $-155.7(2)$ \\
\hline $\mathrm{C} 4-\mathrm{C} 3-\mathrm{C} 8-\mathrm{C} 9$ & $163.3(3)$ & $\mathrm{C} 15-\mathrm{O} 17-\mathrm{C} 18-\mathrm{C} 20$ & $82.1(3)$ \\
\hline $\mathrm{C} 4-\mathrm{C} 5-\mathrm{C} 6-\mathrm{C} 7$ & $-4.2(4)$ & $\mathrm{O} 16-\mathrm{C} 15-\mathrm{O} 17-\mathrm{C} 18$ & $2.6(4)$ \\
\hline $\mathrm{C} 4-\mathrm{C} 5-\mathrm{C} 6-\mathrm{O} 13$ & $175.7(3)$ & $\mathrm{N} 22-\mathrm{C} 23-\mathrm{C} 24-\mathrm{C} 25$ & $0.0(4)$ \\
\hline
\end{tabular}




$\begin{array}{llll}\mathrm{C} 4-\mathrm{C} 5-\mathrm{O} 11-\mathrm{C} 12 & 7.4(4) & \mathrm{C} 23-\mathrm{N} 22-\mathrm{C} 27-\mathrm{C} 26 & 0.1(4) \\ \mathrm{C} 5-\mathrm{C} 6-\mathrm{C} 7-\mathrm{C} 2 & 2.7(4) & \mathrm{C} 23-\mathrm{C} 24-\mathrm{C} 25-\mathrm{C} 26 & 0.2(4) \\ \mathrm{C} 5-\mathrm{C} 6-\mathrm{O} 13-\mathrm{C} 14 & -156.9(3) & \mathrm{C} 24-\mathrm{C} 25-\mathrm{C} 26-\mathrm{C} 27 & -0.2(4) \\ \mathrm{C} 6-\mathrm{C} 5-\mathrm{O} 11-\mathrm{C} 12 & -174.1(2) & \mathrm{C} 25-\mathrm{C} 26-\mathrm{C} 27-\mathrm{N} 22 & 0.1(5) \\ \mathrm{C} 6-\mathrm{O} 13-\mathrm{C} 14-\mathrm{C} 15 & -88.0(3) & \mathrm{C} 27-\mathrm{N} 22-\mathrm{C} 23-\mathrm{C} 24 & -0.1(4)\end{array}$

Hydrogen-bond geometry $\left(\AA,{ }^{\circ}\right)$

$\mathrm{Cg} 5$ is the centroid of the $\mathrm{C} 2-\mathrm{C} 7$ phenyl ring.

\begin{tabular}{lllll}
\hline$D-\mathrm{H} \cdots A$ & $D-\mathrm{H}$ & $\mathrm{H} \cdots A$ & $D \cdots A$ & $D-\mathrm{H} \cdots A$ \\
\hline $\mathrm{C} 12-\mathrm{H} 12 B \cdots \mathrm{O} 16^{\mathrm{i}}$ & 0.98 & 2.42 & $3.354(3)$ & 160 \\
$\mathrm{C} 14-\mathrm{H} 14 A \cdots \mathrm{O} 11^{\mathrm{ii}}$ & 0.99 & 2.31 & $3.266(3)$ & 161 \\
$\mathrm{C} 14-\mathrm{H} 14 A \cdots \mathrm{O} 13^{\mathrm{ii}}$ & 0.99 & 2.56 & $3.330(4)$ & 134 \\
$\mathrm{C} 20-\mathrm{H} 20 B \cdots C g 5^{\mathrm{iii}}$ & 0.98 & 2.93 & $3.586(3)$ & 125 \\
$\mathrm{C} 26-\mathrm{H} 26 \cdots \mathrm{Cg}^{\mathrm{ii}}$ & 0.95 & 2.88 & $3.736(3)$ & 150 \\
\hline
\end{tabular}

Symmetry codes: (i) $x-1, y, z$; (ii) $-x+2,-y,-z+2$; (iii) $x+1, y, z$; (iv) $-x+2,-y+1,-z+1$.

( $\eta^{2}$-2-Allyl-4-methoxy-5-\{[(propan-2-yloxy) carbonyl] methoxy\}phenyl- $\left.\kappa C^{1}\right)$ chlorido(4-methylpyridine-

$\kappa N$ )platinum(II) (II)

\section{Crystal data}

$\left[\mathrm{Pt}\left(\mathrm{C}_{15} \mathrm{H}_{19} \mathrm{O}_{4}\right) \mathrm{Cl}\left(\mathrm{C}_{6} \mathrm{H}_{7} \mathrm{~N}\right)\right]$

$M_{r}=586.97$

Triclinic, $P \overline{1}$

$a=8.36089(15) \AA$

$b=9.12717(16) \AA$

$c=14.5582(3) \AA$

$\alpha=94.9089(15)^{\circ}$

$\beta=102.2766(16)^{\circ}$

$\gamma=100.4541(15)^{\circ}$

$V=1058.58(3) \AA^{3}$

\section{Data collection}

Rigaku Oxford Diffraction SuperNova, Single source at offset/far, Eos

diffractometer

Radiation source: SuperNova (Mo) X-ray

Source

Mirror monochromator

Detector resolution: 15.9631 pixels $\mathrm{mm}^{-1}$

$\omega$ scans

Absorption correction: multi-scan

CrysAlisPro (Rigaku OD, 2018)

Refinement

Refinement on $F^{2}$

Least-squares matrix: full

$R\left[F^{2}>2 \sigma\left(F^{2}\right)\right]=0.013$

$w R\left(F^{2}\right)=0.033$

$S=1.12$

4327 reflections

257 parameters
$Z=2$

$F(000)=572$

$D_{\mathrm{x}}=1.841 \mathrm{Mg} \mathrm{m}^{-3}$

Mo $K \alpha$ radiation, $\lambda=0.71073 \AA$

Cell parameters from 35745 reflections

$\theta=3.2-29.0^{\circ}$

$\mu=6.78 \mathrm{~mm}^{-1}$

$T=100 \mathrm{~K}$

Needle, colourless

$0.25 \times 0.2 \times 0.2 \mathrm{~mm}$

$T_{\min }=0.671, T_{\max }=1.000$

43513 measured reflections

4327 independent reflections

4252 reflections with $I>2 \sigma(I)$

$R_{\text {int }}=0.036$

$\theta_{\text {max }}=26.4^{\circ}, \theta_{\min }=2.9^{\circ}$

$h=-10 \rightarrow 10$

$k=-11 \rightarrow 11$

$l=-18 \rightarrow 18$

0 restraints

Primary atom site location: structure-invariant direct methods

Hydrogen site location: inferred from neighbouring sites

$\mathrm{H}$-atom parameters constrained 
$w=1 /\left[\sigma^{2}\left(F_{\mathrm{o}}^{2}\right)+(0.015 P)^{2}+0.659 P\right]$

where $P=\left(F_{\mathrm{o}}^{2}+2 F_{\mathrm{c}}^{2}\right) / 3$

$(\Delta / \sigma)_{\max }=0.005$

$$
\Delta \rho_{\max }=0.38 \text { e } \AA^{-3}
$$

Special details

Geometry. All esds (except the esd in the dihedral angle between two 1.s. planes) are estimated using the full covariance matrix. The cell esds are taken into account individually in the estimation of esds in distances, angles and torsion angles; correlations between esds in cell parameters are only used when they are defined by crystal symmetry. An approximate (isotropic) treatment of cell esds is used for estimating esds involving 1.s. planes.

Fractional atomic coordinates and isotropic or equivalent isotropic displacement parameters $\left(\AA^{2}\right)$

\begin{tabular}{|c|c|c|c|c|}
\hline & $x$ & $y$ & $z$ & $U_{\text {iso }} * / U_{\text {eq }}$ \\
\hline Pt1 & $1.00923(2)$ & $0.54971(2)$ & $0.69268(2)$ & 0.00989 (3) \\
\hline $\mathrm{C} 2$ & $0.9207(3)$ & $0.3594(2)$ & $0.74088(15)$ & $0.0119(4)$ \\
\hline $\mathrm{C} 3$ & $0.7507(3)$ & $0.2995(2)$ & $0.70567(15)$ & $0.0126(4)$ \\
\hline $\mathrm{C} 4$ & $0.6771(3)$ & $0.1616(2)$ & $0.72883(15)$ & $0.0122(4)$ \\
\hline $\mathrm{H} 4$ & 0.562025 & 0.120185 & 0.702254 & $0.015^{*}$ \\
\hline $\mathrm{C} 5$ & $0.7718(3)$ & $0.0851(2)$ & $0.79059(15)$ & $0.0119(4)$ \\
\hline $\mathrm{C} 6$ & $0.9409(3)$ & $0.1511(2)$ & $0.83258(15)$ & $0.0124(4)$ \\
\hline $\mathrm{C} 7$ & $1.0146(3)$ & $0.2846(2)$ & $0.80607(15)$ & $0.0126(4)$ \\
\hline $\mathrm{H} 7$ & 1.129836 & 0.325802 & 0.832241 & $0.015^{*}$ \\
\hline $\mathrm{C} 8$ & $0.6526(3)$ & $0.3880(2)$ & $0.63896(16)$ & $0.0163(4)$ \\
\hline $\mathrm{H} 8 \mathrm{~A}$ & 0.632773 & 0.340077 & 0.572804 & $0.020^{*}$ \\
\hline H8B & 0.542682 & 0.387817 & 0.654178 & $0.020^{*}$ \\
\hline $\mathrm{C} 9$ & $0.7486(3)$ & $0.5483(2)$ & $0.64848(17)$ & $0.0176(5)$ \\
\hline $\mathrm{H} 9$ & 0.764409 & 0.589874 & 0.592650 & $0.021 *$ \\
\hline $\mathrm{C} 10$ & $0.8151(3)$ & $0.6376(3)$ & $0.73626(18)$ & $0.0202(5)$ \\
\hline $\mathrm{H} 10 \mathrm{~A}$ & 0.800671 & 0.598144 & 0.792944 & $0.024 *$ \\
\hline H10B & 0.874500 & 0.737636 & 0.739110 & $0.024 *$ \\
\hline O11 & $0.71319(18)$ & $-0.05067(16)$ & $0.81844(11)$ & $0.0144(3)$ \\
\hline $\mathrm{C} 12$ & $0.5483(3)$ & $-0.1295(2)$ & $0.76921(16)$ & $0.0164(4)$ \\
\hline $\mathrm{H} 12 \mathrm{~A}$ & 0.542265 & -0.144001 & 0.700981 & $0.025^{*}$ \\
\hline H12B & 0.466172 & -0.070730 & 0.781434 & $0.025^{*}$ \\
\hline $\mathrm{H} 12 \mathrm{C}$ & 0.524050 & -0.227516 & 0.791625 & $0.025^{*}$ \\
\hline $\mathrm{O} 13$ & $1.02076(19)$ & $0.07216(17)$ & $0.89891(11)$ & $0.0171(3)$ \\
\hline $\mathrm{C} 14$ & $1.1632(3)$ & $0.1545(2)$ & $0.96761(15)$ & $0.0155(4)$ \\
\hline $\mathrm{H} 14 \mathrm{~A}$ & 1.173924 & 0.106448 & 1.026448 & $0.019^{*}$ \\
\hline H14B & 1.147068 & 0.257814 & 0.982931 & $0.019^{*}$ \\
\hline $\mathrm{C} 15$ & $1.3235(3)$ & $0.1620(2)$ & $0.93298(15)$ & $0.0134(4)$ \\
\hline O16 & $1.3310(2)$ & $0.10801(18)$ & $0.85616(11)$ & $0.0201(3)$ \\
\hline O17 & $1.45437(18)$ & $0.23658(17)$ & $1.00086(10)$ & $0.0160(3)$ \\
\hline $\mathrm{C} 18$ & $1.6184(3)$ & $0.2570(3)$ & $0.97671(16)$ & $0.0165(4)$ \\
\hline H18 & 1.623467 & 0.166368 & 0.934555 & $0.020^{*}$ \\
\hline C19 & $1.7466(3)$ & $0.2720(3)$ & $1.06927(18)$ & $0.0277(6)$ \\
\hline H19A & 1.858792 & 0.285300 & 1.056968 & $0.042^{*}$ \\
\hline H19B & 1.724572 & 0.181059 & 1.099656 & $0.042 *$ \\
\hline $\mathrm{H} 19 \mathrm{C}$ & 1.739589 & 0.359312 & 1.111216 & $0.042 *$ \\
\hline $\mathrm{C} 20$ & $1.6403(3)$ & $0.3939(3)$ & $0.92522(18)$ & $0.0239(5)$ \\
\hline
\end{tabular}




\begin{tabular}{lllll}
$\mathrm{H} 20 \mathrm{~A}$ & 1.637086 & 0.483010 & 0.966895 & $0.036^{*}$ \\
$\mathrm{H} 20 \mathrm{~B}$ & 1.549879 & 0.379957 & 0.868030 & $0.036^{*}$ \\
$\mathrm{H} 20 \mathrm{C}$ & 1.748194 & 0.407443 & 0.907410 & $0.036^{*}$ \\
$\mathrm{C} 21$ & $1.26067(6)$ & $0.48086(6)$ & $0.68589(4)$ & $0.01889(11)$ \\
$\mathrm{N} 22$ & $1.0906(2)$ & $0.74891(19)$ & $0.63327(13)$ & $0.0129(4)$ \\
$\mathrm{C} 23$ & $1.1236(3)$ & $0.8889(2)$ & $0.68027(15)$ & $0.0135(4)$ \\
$\mathrm{H} 23$ & 1.107292 & 0.901003 & 0.742873 & $0.016^{*}$ \\
$\mathrm{C} 24$ & $1.1802(3)$ & $1.0157(2)$ & $0.64104(15)$ & $0.0147(4)$ \\
$\mathrm{H} 24$ & 1.203176 & 1.112415 & 0.676689 & $0.018^{*}$ \\
$\mathrm{C} 25$ & $1.2034(3)$ & $1.0004(2)$ & $0.54849(16)$ & $0.0146(4)$ \\
$\mathrm{C} 26$ & $1.1654(3)$ & $0.8558(2)$ & $0.49975(16)$ & $0.0175(5)$ \\
$\mathrm{H} 26$ & 1.176376 & 0.840720 & 0.436193 & $0.021^{*}$ \\
$\mathrm{C} 27$ & $1.1117(3)$ & $0.7344(2)$ & $0.54383(16)$ & $0.0168(4)$ \\
$\mathrm{H} 27$ & 1.088589 & 0.636514 & 0.509765 & $0.020^{*}$ \\
$\mathrm{C} 28$ & $1.2680(3)$ & $1.1334(3)$ & $0.50278(17)$ & $0.0195(5)$ \\
$\mathrm{H} 28 \mathrm{~A}$ & 1.256524 & 1.225818 & 0.538017 & $0.029^{*}$ \\
$\mathrm{H} 28 \mathrm{~B}$ & 1.203488 & 1.123624 & 0.437162 & $0.029^{*}$ \\
$\mathrm{H} 28 \mathrm{C}$ & 1.386158 & 1.137376 & 0.503370 & $0.029^{*}$ \\
& & & & \\
\hline
\end{tabular}

Atomic displacement parameters $\left(\AA^{2}\right)$

\begin{tabular}{lllllll}
\hline & $U^{11}$ & $U^{22}$ & $U^{33}$ & $U^{12}$ & $U^{13}$ & $U^{23}$ \\
\hline Pt1 & $0.00854(5)$ & $0.00956(5)$ & $0.01200(5)$ & $0.00219(3)$ & $0.00279(3)$ & $0.00204(3)$ \\
C2 & $0.0118(10)$ & $0.0109(10)$ & $0.0139(10)$ & $0.0034(8)$ & $0.0048(8)$ & $0.0004(8)$ \\
C3 & $0.0119(10)$ & $0.0141(10)$ & $0.0136(10)$ & $0.0058(8)$ & $0.0038(8)$ & $0.0023(8)$ \\
C4 & $0.0073(9)$ & $0.0153(10)$ & $0.0139(10)$ & $0.0024(8)$ & $0.0027(8)$ & $0.0005(8)$ \\
C5 & $0.0113(10)$ & $0.0112(10)$ & $0.0140(10)$ & $0.0011(8)$ & $0.0055(8)$ & $0.0014(8)$ \\
C6 & $0.0111(10)$ & $0.0137(10)$ & $0.0127(10)$ & $0.0044(8)$ & $0.0011(8)$ & $0.0026(8)$ \\
C7 & $0.0090(10)$ & $0.0145(10)$ & $0.0133(10)$ & $0.0016(8)$ & $0.0013(8)$ & $0.0014(8)$ \\
C8 & $0.0108(10)$ & $0.0175(11)$ & $0.0209(12)$ & $0.0042(8)$ & $0.0019(9)$ & $0.0060(9)$ \\
C9 & $0.0095(10)$ & $0.0191(11)$ & $0.0274(13)$ & $0.0063(9)$ & $0.0058(9)$ & $0.0098(9)$ \\
C10 & $0.0173(11)$ & $0.0149(11)$ & $0.0339(14)$ & $0.0066(9)$ & $0.0148(10)$ & $0.0045(10)$ \\
O11 & $0.0092(7)$ & $0.0125(7)$ & $0.0189(8)$ & $-0.0015(6)$ & $-0.0005(6)$ & $0.0044(6)$ \\
C12 & $0.0090(10)$ & $0.0145(10)$ & $0.0229(12)$ & $-0.0014(8)$ & $0.0012(9)$ & $0.0016(9)$ \\
O13 & $0.0109(7)$ & $0.0157(7)$ & $0.0203(8)$ & $-0.0019(6)$ & $-0.0045(6)$ & $0.0080(6)$ \\
C14 & $0.0112(10)$ & $0.0185(11)$ & $0.0138(11)$ & $-0.0009(8)$ & $-0.0010(9)$ & $0.0043(9)$ \\
C15 & $0.0119(10)$ & $0.0112(10)$ & $0.0148(11)$ & $0.0015(8)$ & $-0.0020(8)$ & $0.0032(8)$ \\
O16 & $0.0172(8)$ & $0.0239(8)$ & $0.0160(8)$ & $0.0047(7)$ & $-0.0004(7)$ & $-0.0047(7)$ \\
O17 & $0.0092(7)$ & $0.0218(8)$ & $0.0136(8)$ & $-0.0023(6)$ & $0.0020(6)$ & $-0.0021(6)$ \\
C18 & $0.0105(10)$ & $0.0200(11)$ & $0.0179(11)$ & $-0.0001(8)$ & $0.0051(9)$ & $-0.0017(9)$ \\
C19 & $0.0129(11)$ & $0.0416(15)$ & $0.0245(13)$ & $-0.0005(11)$ & $0.0011(10)$ & $0.0027(11)$ \\
C20 & $0.0212(12)$ & $0.0226(12)$ & $0.0291(14)$ & $0.0001(10)$ & $0.0123(11)$ & $0.0039(10)$ \\
C121 & $0.0115(2)$ & $0.0175(3)$ & $0.0308(3)$ & $0.0050(2)$ & $0.0091(2)$ & $0.0056(2)$ \\
N22 & $0.0119(9)$ & $0.0119(8)$ & $0.0145(9)$ & $0.0022(7)$ & $0.0020(7)$ & $0.0028(7)$ \\
C23 & $0.0118(10)$ & $0.0159(10)$ & $0.0108(10)$ & $0.0021(8)$ & $-0.0007(8)$ & $0.0007(8)$ \\
C24 & $0.0127(10)$ & $0.0142(10)$ & $0.0140(11)$ & $0.0018(8)$ & $-0.0023(8)$ & $-0.0002(8)$ \\
C25 & $0.0088(10)$ & $0.0170(11)$ & $0.0171(11)$ & $0.0021(8)$ & $0.0001(8)$ & $0.0046(9)$ \\
C26 & $0.0199(11)$ & $0.0189(11)$ & $0.0153(11)$ & $0.0044(9)$ & $0.0073(9)$ & $0.0025(9)$ \\
& & & & & &
\end{tabular}




\begin{tabular}{lllllll}
$\mathrm{C} 27$ & $0.0194(11)$ & $0.0143(10)$ & $0.0163(11)$ & $0.0021(9)$ & $0.0059(9)$ & $-0.0021(8)$ \\
$\mathrm{C} 28$ & $0.0195(12)$ & $0.0180(11)$ & $0.0195(12)$ & $0.0008(9)$ & $0.0029(10)$ & $0.0059(9)$ \\
\hline
\end{tabular}

Geometric parameters $\left(A,{ }^{\circ}\right)$

\begin{tabular}{|c|c|c|c|}
\hline $\mathrm{Pt} 1-\mathrm{C} 2$ & $2.005(2)$ & $\mathrm{C} 14-\mathrm{H} 14 \mathrm{~B}$ & 0.9900 \\
\hline Pt1-C9 & $2.134(2)$ & $\mathrm{C} 14-\mathrm{C} 15$ & $1.521(3)$ \\
\hline $\mathrm{Pt} 1-\mathrm{C} 10$ & $2.123(2)$ & $\mathrm{C} 15-\mathrm{O} 16$ & $1.202(3)$ \\
\hline $\mathrm{Pt} 1-\mathrm{C} 21$ & $2.3197(5)$ & $\mathrm{C} 15-\mathrm{O} 17$ & $1.339(3)$ \\
\hline $\mathrm{Pt} 1-\mathrm{N} 22$ & $2.1418(18)$ & $\mathrm{O} 17-\mathrm{C} 18$ & $1.471(3)$ \\
\hline $\mathrm{C} 2-\mathrm{C} 3$ & $1.393(3)$ & $\mathrm{C} 18-\mathrm{H} 18$ & 1.0000 \\
\hline $\mathrm{C} 2-\mathrm{C} 7$ & $1.405(3)$ & $\mathrm{C} 18-\mathrm{C} 19$ & $1.512(3)$ \\
\hline $\mathrm{C} 3-\mathrm{C} 4$ & $1.399(3)$ & $\mathrm{C} 18-\mathrm{C} 20$ & $1.514(3)$ \\
\hline $\mathrm{C} 3-\mathrm{C} 8$ & $1.518(3)$ & C19-H19A & 0.9800 \\
\hline $\mathrm{C} 4-\mathrm{H} 4$ & 0.9500 & C19-H19B & 0.9800 \\
\hline $\mathrm{C} 4-\mathrm{C} 5$ & $1.388(3)$ & $\mathrm{C} 19-\mathrm{H} 19 \mathrm{C}$ & 0.9800 \\
\hline $\mathrm{C} 5-\mathrm{C} 6$ & $1.413(3)$ & $\mathrm{C} 20-\mathrm{H} 20 \mathrm{~A}$ & 0.9800 \\
\hline $\mathrm{C} 5-\mathrm{O} 11$ & $1.375(2)$ & $\mathrm{C} 20-\mathrm{H} 20 \mathrm{~B}$ & 0.9800 \\
\hline $\mathrm{C} 6-\mathrm{C} 7$ & $1.387(3)$ & $\mathrm{C} 20-\mathrm{H} 20 \mathrm{C}$ & 0.9800 \\
\hline $\mathrm{C} 6-\mathrm{O} 13$ & $1.384(2)$ & $\mathrm{N} 22-\mathrm{C} 23$ & $1.348(3)$ \\
\hline $\mathrm{C} 7-\mathrm{H} 7$ & 0.9500 & $\mathrm{~N} 22-\mathrm{C} 27$ & $1.349(3)$ \\
\hline $\mathrm{C} 8-\mathrm{H} 8 \mathrm{~A}$ & 0.9900 & $\mathrm{C} 23-\mathrm{H} 23$ & 0.9500 \\
\hline $\mathrm{C} 8-\mathrm{H} 8 \mathrm{~B}$ & 0.9900 & $\mathrm{C} 23-\mathrm{C} 24$ & $1.386(3)$ \\
\hline $\mathrm{C} 8-\mathrm{C} 9$ & $1.515(3)$ & $\mathrm{C} 24-\mathrm{H} 24$ & 0.9500 \\
\hline $\mathrm{C} 9-\mathrm{H} 9$ & 0.9500 & $\mathrm{C} 24-\mathrm{C} 25$ & $1.400(3)$ \\
\hline $\mathrm{C} 9-\mathrm{C} 10$ & $1.401(3)$ & $\mathrm{C} 25-\mathrm{C} 26$ & $1.392(3)$ \\
\hline $\mathrm{C} 10-\mathrm{H} 10 \mathrm{~A}$ & 0.9500 & $\mathrm{C} 25-\mathrm{C} 28$ & $1.503(3)$ \\
\hline $\mathrm{C} 10-\mathrm{H} 10 \mathrm{~B}$ & 0.9500 & $\mathrm{C} 26-\mathrm{H} 26$ & 0.9500 \\
\hline $\mathrm{O} 11-\mathrm{C} 12$ & $1.436(2)$ & $\mathrm{C} 26-\mathrm{C} 27$ & $1.380(3)$ \\
\hline $\mathrm{C} 12-\mathrm{H} 12 \mathrm{~A}$ & 0.9800 & $\mathrm{C} 27-\mathrm{H} 27$ & 0.9500 \\
\hline $\mathrm{C} 12-\mathrm{H} 12 \mathrm{~B}$ & 0.9800 & $\mathrm{C} 28-\mathrm{H} 28 \mathrm{~A}$ & 0.9800 \\
\hline $\mathrm{C} 12-\mathrm{H} 12 \mathrm{C}$ & 0.9800 & $\mathrm{C} 28-\mathrm{H} 28 \mathrm{~B}$ & 0.9800 \\
\hline $\mathrm{O} 13-\mathrm{C} 14$ & $1.423(3)$ & $\mathrm{C} 28-\mathrm{H} 28 \mathrm{C}$ & 0.9800 \\
\hline C14-H14A & 0.9900 & & \\
\hline $\mathrm{C} 2-\mathrm{Pt} 1-\mathrm{C} 9$ & $81.60(8)$ & $\mathrm{C} 6-\mathrm{O} 13-\mathrm{C} 14$ & $117.11(16)$ \\
\hline $\mathrm{C} 2-\mathrm{Pt} 1-\mathrm{C} 10$ & $86.78(9)$ & $\mathrm{O} 13-\mathrm{C} 14-\mathrm{H} 14 \mathrm{~A}$ & 109.2 \\
\hline $\mathrm{C} 2-\mathrm{Pt} 1-\mathrm{Cl} 21$ & $93.24(6)$ & $\mathrm{O} 13-\mathrm{C} 14-\mathrm{H} 14 \mathrm{~B}$ & 109.2 \\
\hline $\mathrm{C} 2-\mathrm{Pt} 1-\mathrm{N} 22$ & $176.29(7)$ & $\mathrm{O} 13-\mathrm{C} 14-\mathrm{C} 15$ & $112.13(18)$ \\
\hline $\mathrm{C} 9-\mathrm{Pt} 1-\mathrm{Cl} 21$ & $156.66(7)$ & $\mathrm{H} 14 \mathrm{~A}-\mathrm{C} 14-\mathrm{H} 14 \mathrm{~B}$ & 107.9 \\
\hline C9-Pt1-N22 & $95.50(8)$ & $\mathrm{C} 15-\mathrm{C} 14-\mathrm{H} 14 \mathrm{~A}$ & 109.2 \\
\hline $\mathrm{C} 10-\mathrm{Pt} 1-\mathrm{C} 9$ & $38.42(9)$ & $\mathrm{C} 15-\mathrm{C} 14-\mathrm{H} 14 \mathrm{~B}$ & 109.2 \\
\hline $\mathrm{C} 10-\mathrm{Pt} 1-\mathrm{Cl} 21$ & $164.63(7)$ & $\mathrm{O} 16-\mathrm{C} 15-\mathrm{C} 14$ & $124.81(19)$ \\
\hline $\mathrm{C} 10-\mathrm{Pt} 1-\mathrm{N} 22$ & $92.37(8)$ & $\mathrm{O} 16-\mathrm{C} 15-\mathrm{O} 17$ & $125.2(2)$ \\
\hline $\mathrm{N} 22-\mathrm{Pt} 1-\mathrm{Cl} 21$ & $88.53(5)$ & $\mathrm{O} 17-\mathrm{C} 15-\mathrm{C} 14$ & $109.96(18)$ \\
\hline $\mathrm{C} 3-\mathrm{C} 2-\mathrm{Pt} 1$ & $115.65(15)$ & $\mathrm{C} 15-\mathrm{O} 17-\mathrm{C} 18$ & $116.30(16)$ \\
\hline $\mathrm{C} 3-\mathrm{C} 2-\mathrm{C} 7$ & $118.71(19)$ & $\mathrm{O} 17-\mathrm{C} 18-\mathrm{H} 18$ & 109.5 \\
\hline $\mathrm{C} 7-\mathrm{C} 2-\mathrm{Pt} 1$ & $125.64(16)$ & $\mathrm{O} 17-\mathrm{C} 18-\mathrm{C} 19$ & $106.23(18)$ \\
\hline
\end{tabular}




\begin{tabular}{|c|c|c|c|}
\hline $\mathrm{C} 2-\mathrm{C} 3-\mathrm{C} 4$ & $120.89(19)$ & $\mathrm{O} 17-\mathrm{C} 18-\mathrm{C} 20$ & $108.67(18)$ \\
\hline $\mathrm{C} 2-\mathrm{C} 3-\mathrm{C} 8$ & $116.84(19)$ & $\mathrm{C} 19-\mathrm{C} 18-\mathrm{H} 18$ & 109.5 \\
\hline $\mathrm{C} 4-\mathrm{C} 3-\mathrm{C} 8$ & $122.23(19)$ & $\mathrm{C} 19-\mathrm{C} 18-\mathrm{C} 20$ & $113.4(2)$ \\
\hline $\mathrm{C} 3-\mathrm{C} 4-\mathrm{H} 4$ & 119.9 & $\mathrm{C} 20-\mathrm{C} 18-\mathrm{H} 18$ & 109.5 \\
\hline $\mathrm{C} 5-\mathrm{C} 4-\mathrm{C} 3$ & $120.14(19)$ & $\mathrm{C} 18-\mathrm{C} 19-\mathrm{H} 19 \mathrm{~A}$ & 109.5 \\
\hline $\mathrm{C} 5-\mathrm{C} 4-\mathrm{H} 4$ & 119.9 & $\mathrm{C} 18-\mathrm{C} 19-\mathrm{H} 19 \mathrm{~B}$ & 109.5 \\
\hline $\mathrm{C} 4-\mathrm{C} 5-\mathrm{C} 6$ & $119.24(19)$ & $\mathrm{C} 18-\mathrm{C} 19-\mathrm{H} 19 \mathrm{C}$ & 109.5 \\
\hline $\mathrm{O} 11-\mathrm{C} 5-\mathrm{C} 4$ & $125.30(19)$ & H19A-C19-H19B & 109.5 \\
\hline $\mathrm{O} 11-\mathrm{C} 5-\mathrm{C} 6$ & $115.42(18)$ & $\mathrm{H} 19 \mathrm{~A}-\mathrm{C} 19-\mathrm{H} 19 \mathrm{C}$ & 109.5 \\
\hline $\mathrm{C} 7-\mathrm{C} 6-\mathrm{C} 5$ & $120.07(19)$ & $\mathrm{H} 19 \mathrm{~B}-\mathrm{C} 19-\mathrm{H} 19 \mathrm{C}$ & 109.5 \\
\hline $\mathrm{O} 13-\mathrm{C} 6-\mathrm{C} 5$ & $114.89(18)$ & $\mathrm{C} 18-\mathrm{C} 20-\mathrm{H} 20 \mathrm{~A}$ & 109.5 \\
\hline $\mathrm{O} 13-\mathrm{C} 6-\mathrm{C} 7$ & $125.04(19)$ & $\mathrm{C} 18-\mathrm{C} 20-\mathrm{H} 20 \mathrm{~B}$ & 109.5 \\
\hline $\mathrm{C} 2-\mathrm{C} 7-\mathrm{H} 7$ & 119.7 & $\mathrm{C} 18-\mathrm{C} 20-\mathrm{H} 20 \mathrm{C}$ & 109.5 \\
\hline $\mathrm{C} 6-\mathrm{C} 7-\mathrm{C} 2$ & $120.66(19)$ & $\mathrm{H} 20 \mathrm{~A}-\mathrm{C} 20-\mathrm{H} 20 \mathrm{~B}$ & 109.5 \\
\hline $\mathrm{C} 6-\mathrm{C} 7-\mathrm{H} 7$ & 119.7 & $\mathrm{H} 20 \mathrm{~A}-\mathrm{C} 20-\mathrm{H} 20 \mathrm{C}$ & 109.5 \\
\hline $\mathrm{C} 3-\mathrm{C} 8-\mathrm{H} 8 \mathrm{~A}$ & 109.6 & $\mathrm{H} 20 \mathrm{~B}-\mathrm{C} 20-\mathrm{H} 20 \mathrm{C}$ & 109.5 \\
\hline $\mathrm{C} 3-\mathrm{C} 8-\mathrm{H} 8 \mathrm{~B}$ & 109.6 & $\mathrm{C} 23-\mathrm{N} 22-\mathrm{Pt} 1$ & $123.87(15)$ \\
\hline $\mathrm{H} 8 \mathrm{~A}-\mathrm{C} 8-\mathrm{H} 8 \mathrm{~B}$ & 108.2 & $\mathrm{C} 23-\mathrm{N} 22-\mathrm{C} 27$ & $117.67(18)$ \\
\hline $\mathrm{C} 9-\mathrm{C} 8-\mathrm{C} 3$ & $110.11(18)$ & $\mathrm{C} 27-\mathrm{N} 22-\mathrm{Pt} 1$ & $118.46(14)$ \\
\hline $\mathrm{C} 9-\mathrm{C} 8-\mathrm{H} 8 \mathrm{~A}$ & 109.6 & $\mathrm{~N} 22-\mathrm{C} 23-\mathrm{H} 23$ & 118.6 \\
\hline $\mathrm{C} 9-\mathrm{C} 8-\mathrm{H} 8 \mathrm{~B}$ & 109.6 & $\mathrm{~N} 22-\mathrm{C} 23-\mathrm{C} 24$ & $122.7(2)$ \\
\hline $\mathrm{Pt} 1-\mathrm{C} 9-\mathrm{H} 9$ & 91.2 & $\mathrm{C} 24-\mathrm{C} 23-\mathrm{H} 23$ & 118.6 \\
\hline $\mathrm{C} 8-\mathrm{C} 9-\mathrm{Pt} 1$ & $108.44(14)$ & $\mathrm{C} 23-\mathrm{C} 24-\mathrm{H} 24$ & 120.2 \\
\hline $\mathrm{C} 8-\mathrm{C} 9-\mathrm{H} 9$ & 118.7 & $\mathrm{C} 23-\mathrm{C} 24-\mathrm{C} 25$ & $119.5(2)$ \\
\hline $\mathrm{C} 10-\mathrm{C} 9-\mathrm{Pt} 1$ & $70.35(12)$ & $\mathrm{C} 25-\mathrm{C} 24-\mathrm{H} 24$ & 120.2 \\
\hline $\mathrm{C} 10-\mathrm{C} 9-\mathrm{C} 8$ & $122.7(2)$ & $\mathrm{C} 24-\mathrm{C} 25-\mathrm{C} 28$ & $122.0(2)$ \\
\hline $\mathrm{C} 10-\mathrm{C} 9-\mathrm{H} 9$ & 118.7 & $\mathrm{C} 26-\mathrm{C} 25-\mathrm{C} 24$ & $117.3(2)$ \\
\hline $\mathrm{Pt} 1-\mathrm{C} 10-\mathrm{H} 10 \mathrm{~A}$ & 107.5 & $\mathrm{C} 26-\mathrm{C} 25-\mathrm{C} 28$ & $120.6(2)$ \\
\hline $\mathrm{Pt} 1-\mathrm{C} 10-\mathrm{H} 10 \mathrm{~B}$ & 91.2 & $\mathrm{C} 25-\mathrm{C} 26-\mathrm{H} 26$ & 120.0 \\
\hline $\mathrm{C} 9-\mathrm{C} 10-\mathrm{Pt} 1$ & $71.23(12)$ & $\mathrm{C} 27-\mathrm{C} 26-\mathrm{C} 25$ & $119.9(2)$ \\
\hline $\mathrm{C} 9-\mathrm{C} 10-\mathrm{H} 10 \mathrm{~A}$ & 120.0 & $\mathrm{C} 27-\mathrm{C} 26-\mathrm{H} 26$ & 120.0 \\
\hline $\mathrm{C} 9-\mathrm{C} 10-\mathrm{H} 10 \mathrm{~B}$ & 120.0 & $\mathrm{~N} 22-\mathrm{C} 27-\mathrm{C} 26$ & $122.8(2)$ \\
\hline $\mathrm{H} 10 \mathrm{~A}-\mathrm{C} 10-\mathrm{H} 10 \mathrm{~B}$ & 120.0 & $\mathrm{~N} 22-\mathrm{C} 27-\mathrm{H} 27$ & 118.6 \\
\hline $\mathrm{C} 5-\mathrm{O} 11-\mathrm{C} 12$ & $116.96(16)$ & $\mathrm{C} 26-\mathrm{C} 27-\mathrm{H} 27$ & 118.6 \\
\hline $\mathrm{O} 11-\mathrm{C} 12-\mathrm{H} 12 \mathrm{~A}$ & 109.5 & $\mathrm{C} 25-\mathrm{C} 28-\mathrm{H} 28 \mathrm{~A}$ & 109.5 \\
\hline $\mathrm{O} 11-\mathrm{C} 12-\mathrm{H} 12 \mathrm{~B}$ & 109.5 & $\mathrm{C} 25-\mathrm{C} 28-\mathrm{H} 28 \mathrm{~B}$ & 109.5 \\
\hline $\mathrm{O} 11-\mathrm{C} 12-\mathrm{H} 12 \mathrm{C}$ & 109.5 & $\mathrm{C} 25-\mathrm{C} 28-\mathrm{H} 28 \mathrm{C}$ & 109.5 \\
\hline $\mathrm{H} 12 \mathrm{~A}-\mathrm{C} 12-\mathrm{H} 12 \mathrm{~B}$ & 109.5 & $\mathrm{H} 28 \mathrm{~A}-\mathrm{C} 28-\mathrm{H} 28 \mathrm{~B}$ & 109.5 \\
\hline $\mathrm{H} 12 \mathrm{~A}-\mathrm{C} 12-\mathrm{H} 12 \mathrm{C}$ & 109.5 & $\mathrm{H} 28 \mathrm{~A}-\mathrm{C} 28-\mathrm{H} 28 \mathrm{C}$ & 109.5 \\
\hline $\mathrm{H} 12 \mathrm{~B}-\mathrm{C} 12-\mathrm{H} 12 \mathrm{C}$ & 109.5 & $\mathrm{H} 28 \mathrm{~B}-\mathrm{C} 28-\mathrm{H} 28 \mathrm{C}$ & 109.5 \\
\hline $\mathrm{Pt} 1-\mathrm{C} 2-\mathrm{C} 3-\mathrm{C} 4$ & $175.43(16)$ & $\mathrm{C} 7-\mathrm{C} 2-\mathrm{C} 3-\mathrm{C} 8$ & $177.26(19)$ \\
\hline $\mathrm{Pt} 1-\mathrm{C} 2-\mathrm{C} 3-\mathrm{C} 8$ & $-2.5(2)$ & $\mathrm{C} 7-\mathrm{C} 6-\mathrm{O} 13-\mathrm{C} 14$ & $23.4(3)$ \\
\hline $\mathrm{Pt} 1-\mathrm{C} 2-\mathrm{C} 7-\mathrm{C} 6$ & $-178.28(16)$ & $\mathrm{C} 8-\mathrm{C} 3-\mathrm{C} 4-\mathrm{C} 5$ & $-179.68(19)$ \\
\hline $\mathrm{Pt} 1-\mathrm{N} 22-\mathrm{C} 23-\mathrm{C} 24$ & $178.30(15)$ & $\mathrm{C} 8-\mathrm{C} 9-\mathrm{C} 10-\mathrm{Pt} 1$ & $99.79(19)$ \\
\hline $\mathrm{Pt} 1-\mathrm{N} 22-\mathrm{C} 27-\mathrm{C} 26$ & $-179.30(17)$ & $\mathrm{O} 11-\mathrm{C} 5-\mathrm{C} 6-\mathrm{C} 7$ & $176.98(18)$ \\
\hline $\mathrm{C} 2-\mathrm{C} 3-\mathrm{C} 4-\mathrm{C} 5$ & $2.5(3)$ & $\mathrm{O} 11-\mathrm{C} 5-\mathrm{C} 6-\mathrm{O} 13$ & $-2.7(3)$ \\
\hline $\mathrm{C} 2-\mathrm{C} 3-\mathrm{C} 8-\mathrm{C} 9$ & $-17.9(3)$ & $\mathrm{O} 13-\mathrm{C} 6-\mathrm{C} 7-\mathrm{C} 2$ & $-177.25(19)$ \\
\hline
\end{tabular}




\begin{tabular}{ll}
$\mathrm{C} 3-\mathrm{C} 2-\mathrm{C} 7-\mathrm{C} 6$ & $2.0(3)$ \\
$\mathrm{C} 3-\mathrm{C} 4-\mathrm{C} 5-\mathrm{C} 6$ & $2.6(3)$ \\
$\mathrm{C} 3-\mathrm{C} 4-\mathrm{C} 5-\mathrm{O} 11$ & $179.99(19)$ \\
$\mathrm{C} 3-\mathrm{C} 8-\mathrm{C} 9-\mathrm{P} 1$ & $27.8(2)$ \\
$\mathrm{C} 3-\mathrm{C} 8-\mathrm{C} 9-\mathrm{C} 10$ & $-50.2(3)$ \\
$\mathrm{C} 4-\mathrm{C} 3-\mathrm{C} 8-\mathrm{C} 9$ & $164.2(2)$ \\
$\mathrm{C} 4-\mathrm{C} 5-\mathrm{C} 6-\mathrm{C} 7$ & $-5.4(3)$ \\
$\mathrm{C} 4-\mathrm{C} 5-\mathrm{C} 6-\mathrm{O} 13$ & $174.91(18)$ \\
$\mathrm{C} 4-\mathrm{C} 5-\mathrm{O} 11-\mathrm{C} 12$ & $9.6(3)$ \\
$\mathrm{C} 5-\mathrm{C} 6-\mathrm{C} 7-\mathrm{C} 2$ & $3.1(3)$ \\
$\mathrm{C} 5-\mathrm{C} 6-\mathrm{O} 13-\mathrm{C} 14$ & $-156.95(19)$ \\
$\mathrm{C} 6-\mathrm{C} 5-\mathrm{O} 11-\mathrm{C} 12$ & $-172.89(18)$ \\
$\mathrm{C} 6-\mathrm{O} 13-\mathrm{C} 14-\mathrm{C} 15$ & $-87.5(2)$ \\
$\mathrm{C} 7-\mathrm{C} 2-\mathrm{C} 3-\mathrm{C} 4$ & $-4.8(3)$ \\
\hline
\end{tabular}

$1.4(3)$

$-178.19(16)$

$-178.25(17)$

$-153.51(19)$

$84.2(2)$

$2.2(3)$

$0.7(3)$

$0.2(3)$

$0.8(3)$

$-178.7(2)$

$-1.7(3)$

$1.3(3)$

$-1.2(3)$

$177.8(2)$

Hydrogen-bond geometry $\left(A,{ }^{\circ}\right)$

$\mathrm{Cg} 5$ is the centroid of the $\mathrm{C} 2-\mathrm{C} 7$ phenyl ring.

\begin{tabular}{lllll}
\hline$D-\mathrm{H} \cdots A$ & $D-\mathrm{H}$ & $\mathrm{H} \cdots A$ & $D \cdots A$ & $D-\mathrm{H} \cdots A$ \\
\hline $\mathrm{C} 12-\mathrm{H} 12 B \cdots \mathrm{O} 16^{\mathrm{i}}$ & 0.98 & 2.45 & $3.397(3)$ & 162 \\
$\mathrm{C} 14-\mathrm{H} 14 A \cdots \mathrm{O} 11^{\mathrm{ii}}$ & 0.99 & 2.39 & $3.341(3)$ & 161 \\
$\mathrm{C} 14-\mathrm{H} 14 A \cdots \mathrm{O} 13^{\mathrm{ii}}$ & 0.99 & 2.57 & $3.351(3)$ & 136 \\
$\mathrm{C} 8-\mathrm{H} 8 B \cdots \mathrm{C} 21^{\mathrm{i}}$ & 0.99 & 2.76 & $3.713(3)$ & 162 \\
$\mathrm{C} 20-\mathrm{H} 20 B \cdots C g 5^{\mathrm{iii}}$ & 0.98 & 2.87 & $3.562(3)$ & 128 \\
$\mathrm{C} 26-\mathrm{H} 26 \cdots C g 5^{\mathrm{iv}}$ & 0.95 & 2.93 & $3.873(3)$ & 171 \\
$\mathrm{C} 28-\mathrm{H} 28 B \cdots C g 4^{\mathrm{v}}$ & 0.98 & 2.87 & $3.425(3)$ & 117 \\
\hline
\end{tabular}

Symmetry codes: (i) $x-1, y, z$; (ii) $-x+2,-y,-z+2$; (iii) $x+1, y, z$; (iv) $-x+2,-y+1,-z+1$; (v) $-x+2,-y+2,-z+1$.

( $\eta^{2}$-2-Allyl-4-methoxy-5-\{[(propan-2-yloxy)carbonyl]methoxy\}phenyl- $\left.\kappa C^{1}\right)$ chlorido(pyridine-4-carboxylic acid-

$\kappa \mathrm{N})$ platinum(II) (III)

\section{Crystal data}

$\left[\mathrm{Pt}\left(\mathrm{C}_{15} \mathrm{H}_{19} \mathrm{O}_{4}\right) \mathrm{Cl}\left(\mathrm{C}_{6} \mathrm{H}_{5} \mathrm{NO}_{2}\right)\right]$

$M_{r}=616.95$

Triclinic, $P \overline{1}$

$a=7.8746(2) \AA$

$b=9.7566(2) \AA$

$c=15.0004(4) \AA$

$\alpha=95.782(2)^{\circ}$

$\beta=102.874(2)^{\circ}$

$\gamma=93.843(2)^{\circ}$

$V=1113.02(5) \AA^{3}$

\section{Data collection}

Rigaku Oxford Diffraction SuperNova, Single source at offset/far, Eos diffractometer

Radiation source: micro-focus sealed X-ray tube, SuperNova (Mo) X-ray Source Mirror monochromator
$Z=2$

$F(000)=600$

$D_{\mathrm{x}}=1.841 \mathrm{Mg} \mathrm{m}^{-3}$

Mo $K \alpha$ radiation, $\lambda=0.71073 \AA$

Cell parameters from 13370 reflections

$\theta=2.8-29.1^{\circ}$

$\mu=6.46 \mathrm{~mm}^{-1}$

$T=100 \mathrm{~K}$

Block, light yellow

$0.4 \times 0.4 \times 0.35 \mathrm{~mm}$

Detector resolution: 15.9631 pixels $\mathrm{mm}^{-1}$

$\omega$ scans

Absorption correction: multi-scan

CrysAlisPro (Rigaku OD, 2018)

$T_{\min }=0.429, T_{\max }=1.000$

22839 measured reflections 
4542 independent reflections

4276 reflections with $I>2 \sigma(I)$

$R_{\text {int }}=0.077$

$\theta_{\max }=26.4^{\circ}, \theta_{\min }=2.7^{\circ}$

\section{Refinement}

Refinement on $F^{2}$

Least-squares matrix: full

$R\left[F^{2}>2 \sigma\left(F^{2}\right)\right]=0.027$

$w R\left(F^{2}\right)=0.068$

$S=1.05$

4542 reflections

275 parameters

27 restraints

$$
\begin{aligned}
& h=-9 \rightarrow 9 \\
& k=-12 \rightarrow 12 \\
& l=-18 \rightarrow 18
\end{aligned}
$$

Primary atom site location: structure-invariant direct methods

Hydrogen site location: inferred from neighbouring sites

$\mathrm{H}$-atom parameters constrained

$w=1 /\left[\sigma^{2}\left(F_{\mathrm{o}}^{2}\right)+(0.0316 P)^{2}+0.7413 P\right]$ where $P=\left(F_{\mathrm{o}}^{2}+2 F_{\mathrm{c}}{ }^{2}\right) / 3$

$(\Delta / \sigma)_{\max }=0.002$

$\Delta \rho_{\max }=1.87{\mathrm{e} \AA^{-3}}^{-3}$

$\Delta \rho_{\min }=-1.77{\mathrm{e} \AA^{-3}}^{-3}$

Special details

Geometry. All esds (except the esd in the dihedral angle between two 1.s. planes) are estimated using the full covariance matrix. The cell esds are taken into account individually in the estimation of esds in distances, angles and torsion angles; correlations between esds in cell parameters are only used when they are defined by crystal symmetry. An approximate

\begin{tabular}{|c|c|c|c|c|}
\hline & $x$ & $y$ & $z$ & $U_{\text {iso }} * / U_{\text {eq }}$ \\
\hline Pt1 & -0.08260 & $0.65313(2)$ & $0.08249(2)$ & $0.01501(7)$ \\
\hline $\mathrm{C} 2$ & $0.0203(5)$ & $0.6097(4)$ & $0.2108(3)$ & $0.0169(8)$ \\
\hline $\mathrm{C} 3$ & $-0.0464(5)$ & $0.4856(4)$ & $0.2343(3)$ & $0.0187(9)$ \\
\hline $\mathrm{C} 4$ & $0.0185(5)$ & $0.4418(4)$ & 0.3205 & $0.0183(8)$ \\
\hline $\mathrm{H} 4$ & -0.028452 & 0.357164 & 0.335382 & $0.022 *$ \\
\hline $\mathrm{C} 5$ & $0.1523(5)$ & $0.5231(4)$ & $0.3843(3)$ & $0.0178(9)$ \\
\hline C6 & $0.2201(5)$ & $0.6479(4)$ & $0.3614(3)$ & $0.0174(8)$ \\
\hline $\mathrm{C} 7$ & $0.1555(5)$ & $0.6914(4)$ & $0.2757(3)$ & $0.0169(8)$ \\
\hline $\mathrm{H} 7$ & 0.202597 & 0.776169 & 0.260973 & $0.020 *$ \\
\hline $\mathrm{C} 8$ & $-0.1822(6)$ & $0.3961(4)$ & $0.1617(3)$ & $0.0217(9)$ \\
\hline H8A & -0.273612 & 0.356019 & 0.189827 & $0.026^{*}$ \\
\hline H8B & -0.127352 & 0.319045 & 0.134342 & $0.026^{*}$ \\
\hline $\mathrm{C} 9$ & $-0.2649(5)$ & $0.4817(4)$ & $0.0868(3)$ & $0.0211(9)$ \\
\hline H9 & -0.269614 & 0.449717 & 0.024284 & $0.025^{*}$ \\
\hline $\mathrm{C} 10$ & $-0.3329(5)$ & $0.6041(5)$ & $0.1073(3)$ & $0.0245(10)$ \\
\hline H10A & -0.329033 & 0.637354 & 0.169486 & $0.029 *$ \\
\hline H10B & -0.383691 & 0.655430 & 0.059143 & $0.029 *$ \\
\hline O11 & $0.2235(4)$ & $0.4935(3)$ & $0.47161(19)$ & $0.0224(7)$ \\
\hline C12 & $0.1579(6)$ & $0.3684(4)$ & $0.4996(3)$ & $0.0239(9)$ \\
\hline $\mathrm{H} 12 \mathrm{~A}$ & 0.211332 & 0.364448 & 0.564733 & $0.036^{*}$ \\
\hline H12B & 0.186894 & 0.288719 & 0.462377 & $0.036^{*}$ \\
\hline $\mathrm{H} 12 \mathrm{C}$ & 0.030620 & 0.366276 & 0.490730 & $0.036^{*}$ \\
\hline O13 & $0.3539(4)$ & 0.7181 & $0.43083(18)$ & $0.0200(6)$ \\
\hline $\mathrm{C} 14$ & $0.4376(6)$ & 0.8428 & $0.4126(3)$ & $0.0219(9)$ \\
\hline H14A & 0.505769 & 0.893802 & 0.471514 & $0.026^{*}$ \\
\hline
\end{tabular}
(isotropic) treatment of cell esds is used for estimating esds involving 1.s. planes.

Fractional atomic coordinates and isotropic or equivalent isotropic displacement parameters $\left(\AA^{2}\right)$ 


$\begin{array}{lllll}\text { H14B } & 0.347315 & 0.901710 & 0.384927 & 0.026^{*} \\ \text { C15 } & 0.5587(6) & 0.8167(4) & 0.3483(3) & 0.0239(10) \\ \text { O16 } & 0.5679(4) & 0.7092(3) & 0.3051(2) & 0.0245(7) \\ \text { O17 } & 0.6589(5) & 0.9325(3) & 0.3507(3) & 0.0485(10) \\ \text { C18 } & 0.7874(8) & 0.9294(6) & 0.2922(5) & 0.0561(14) \\ \text { H18 } & 0.823471 & 0.833482 & 0.284121 & 0.067^{*} \\ \text { C19 } & 0.9440(9) & 1.0257(7) & 0.3460(7) & 0.091(2) \\ \text { H19A } & 0.999568 & 0.985788 & 0.401411 & 0.136^{*} \\ \text { H19B } & 0.905267 & 1.115713 & 0.363746 & 0.136^{*} \\ \text { H19C } & 1.028227 & 1.037723 & 0.307526 & 0.136^{*} \\ \text { C20 } & 0.7028(9) & 0.9695(7) & 0.1996(6) & 0.0701(17) \\ \text { H20A } & 0.678687 & 1.066797 & 0.206270 & 0.105^{*} \\ \text { H20B } & 0.592848 & 0.911267 & 0.174277 & 0.105^{*} \\ \text { H20C } & 0.781548 & 0.956588 & 0.157863 & 0.105^{*} \\ \text { C121 } & 0.16747(13) & 0.78892(10) & 0.07378(7) & 0.0212(2) \\ \text { N22 } & -0.2017(4) & 0.6881(4) & -0.0569(2) & 0.0177(7) \\ \text { C23 } & -0.1998(6) & 0.8167(4) & -0.0836(3) & 0.0208(9) \\ \text { H23 } & -0.146276 & 0.892910 & -0.039469 & 0.025^{*} \\ \text { C24 } & -0.2727(5) & 0.8411(4) & -0.1723(3) & 0.0194(9) \\ \text { H24 } & -0.264964 & 0.932045 & -0.189494 & 0.023^{*} \\ \text { C25 } & -0.3581(5) & 0.7307(4) & -0.2368(3) & 0.0152(8) \\ \text { C26 } & -0.3638(5) & 0.5988(4) & -0.2095(3) & 0.0160(8) \\ \text { H26 } & -0.422090 & 0.521699 & -0.251417 & 0.019^{*} \\ \text { C27 } & -0.2827(5) & 0.5822(4) & -0.1198(3) & 0.0170(8) \\ \text { H27 } & -0.284227 & 0.491574 & -0.101825 & 0.020^{*} \\ \text { C28 } & -0.4300(5) & 0.7530(4) & -0.3353(3) & 0.0173(8) \\ \text { O29 } & -0.3773(4) & 0.8497(3) & -0.3691(2) & 0.0245(7) \\ \text { O30 } & -0.5521(4) & 0.6547(3) & -0.3784(2) & 0.0275(7) \\ \text { H30 } & -0.576378 & 0.661965 & -0.435015 & 0.041^{*} \\ & & & & \end{array}$

Atomic displacement parameters $\left(\AA^{2}\right)$

\begin{tabular}{lllllll}
\hline & $U^{11}$ & $U^{22}$ & $U^{33}$ & $U^{12}$ & $U^{13}$ & $U^{23}$ \\
\hline Pt1 & $0.01412(10)$ & $0.01776(10)$ & $0.00990(10)$ & $-0.00293(7)$ & $-0.00293(6)$ & $0.00194(6)$ \\
C2 & $0.014(2)$ & $0.020(2)$ & $0.014(2)$ & $0.0018(16)$ & $-0.0022(16)$ & $0.0023(16)$ \\
C3 & $0.015(2)$ & $0.023(2)$ & $0.015(2)$ & $-0.0046(17)$ & $0.0008(16)$ & $-0.0015(17)$ \\
C4 & $0.019(2)$ & $0.022(2)$ & $0.013(2)$ & $-0.0014(17)$ & $0.0005(16)$ & $0.0046(16)$ \\
C5 & $0.019(2)$ & $0.023(2)$ & $0.0099(19)$ & $0.0038(17)$ & $-0.0003(16)$ & $0.0039(16)$ \\
C6 & $0.017(2)$ & $0.020(2)$ & $0.012(2)$ & $0.0016(16)$ & $-0.0024(16)$ & $-0.0019(16)$ \\
C7 & $0.017(2)$ & $0.018(2)$ & $0.0115(19)$ & $-0.0026(16)$ & $-0.0020(16)$ & $-0.0017(16)$ \\
C8 & $0.024(2)$ & $0.026(2)$ & $0.012(2)$ & $-0.0062(18)$ & $-0.0012(17)$ & $0.0041(17)$ \\
C9 & $0.016(2)$ & $0.028(2)$ & $0.015(2)$ & $-0.0119(18)$ & $0.0013(17)$ & $0.0022(17)$ \\
C10 & $0.013(2)$ & $0.040(3)$ & $0.019(2)$ & $-0.0039(19)$ & $0.0005(17)$ & $0.0070(19)$ \\
O11 & $0.0249(16)$ & $0.0260(16)$ & $0.0124(15)$ & $-0.0013(13)$ & $-0.0049(12)$ & $0.0070(12)$ \\
C12 & $0.026(2)$ & $0.026(2)$ & $0.020(2)$ & $0.0035(19)$ & $0.0027(18)$ & $0.0083(18)$ \\
O13 & $0.0235(16)$ & $0.0185(14)$ & $0.0110(14)$ & $-0.0055(12)$ & $-0.0084(12)$ & $0.0013(11)$ \\
C14 & $0.026(2)$ & $0.016(2)$ & $0.018(2)$ & $-0.0019(17)$ & $-0.0063(18)$ & $-0.0013(16)$ \\
C15 & $0.018(2)$ & $0.016(2)$ & $0.032(3)$ & $-0.0025(17)$ & $-0.0063(18)$ & $0.0036(18)$
\end{tabular}


supporting information

\begin{tabular}{lllllll} 
O16 & $0.0247(17)$ & $0.0199(15)$ & $0.0252(17)$ & $-0.0025(13)$ & $0.0003(13)$ & $0.0011(13)$ \\
O17 & $0.036(2)$ & $0.0189(17)$ & $0.095(3)$ & $-0.0076(15)$ & $0.034(2)$ & $-0.0052(18)$ \\
C18 & $0.042(3)$ & $0.023(3)$ & $0.111(4)$ & $-0.009(2)$ & $0.045(3)$ & $-0.007(3)$ \\
C19 & $0.045(3)$ & $0.049(4)$ & $0.178(7)$ & $-0.024(3)$ & $0.047(4)$ & $-0.020(4)$ \\
C20 & $0.065(4)$ & $0.047(4)$ & $0.125(4)$ & $0.018(3)$ & $0.067(3)$ & $0.023(3)$ \\
C121 & $0.0205(5)$ & $0.0226(5)$ & $0.0168(5)$ & $-0.0075(4)$ & $-0.0013(4)$ & $0.0031(4)$ \\
N22 & $0.0149(18)$ & $0.0186(17)$ & $0.0158(18)$ & $-0.0037(14)$ & $-0.0022(14)$ & $0.0006(14)$ \\
C23 & $0.024(2)$ & $0.018(2)$ & $0.016(2)$ & $-0.0053(17)$ & $-0.0012(17)$ & $0.0012(17)$ \\
C24 & $0.023(2)$ & $0.0152(19)$ & $0.016(2)$ & $-0.0005(17)$ & $-0.0018(17)$ & $0.0020(16)$ \\
C25 & $0.0131(19)$ & $0.0181(19)$ & $0.013(2)$ & $0.0024(16)$ & $-0.0005(15)$ & $0.0041(16)$ \\
C26 & $0.015(2)$ & $0.0165(19)$ & $0.013(2)$ & $-0.0004(16)$ & $-0.0013(16)$ & $-0.0014(16)$ \\
C27 & $0.018(2)$ & $0.0157(19)$ & $0.014(2)$ & $-0.0015(16)$ & $-0.0020(16)$ & $0.0026(16)$ \\
C28 & $0.0174(18)$ & $0.0186(15)$ & $0.0125(18)$ & $0.0013(12)$ & $-0.0030(14)$ & $0.0010(12)$ \\
O29 & $0.0297(17)$ & $0.0234(14)$ & $0.0165(15)$ & $-0.0021(12)$ & $-0.0035(13)$ & $0.0067(11)$ \\
O30 & $0.0302(17)$ & $0.0320(15)$ & $0.0113(15)$ & $-0.0110(13)$ & $-0.0095(13)$ & $0.0017(12)$ \\
& & & & & & \\
\hline
\end{tabular}

Geometric parameters $\left(A,{ }^{o}\right)$

\begin{tabular}{|c|c|c|c|}
\hline $\mathrm{Pt} 1-\mathrm{C} 2$ & $2.014(4)$ & C14-H14B & 0.9900 \\
\hline $\mathrm{Pt} 1-\mathrm{C} 9$ & $2.146(4)$ & $\mathrm{C} 14-\mathrm{C} 15$ & $1.517(6)$ \\
\hline $\mathrm{Pt} 1-\mathrm{C} 10$ & $2.118(4)$ & $\mathrm{C} 15-\mathrm{O} 16$ & $1.191(5)$ \\
\hline $\mathrm{Pt} 1-\mathrm{Cl} 21$ & $2.3345(10)$ & $\mathrm{C} 15-\mathrm{O} 17$ & $1.327(5)$ \\
\hline Pt1-N22 & $2.164(3)$ & $\mathrm{O} 17-\mathrm{C} 18$ & $1.480(7)$ \\
\hline $\mathrm{C} 2-\mathrm{C} 3$ & $1.396(6)$ & $\mathrm{C} 18-\mathrm{H} 18$ & 1.0000 \\
\hline $\mathrm{C} 2-\mathrm{C} 7$ & $1.409(5)$ & $\mathrm{C} 18-\mathrm{C} 19$ & $1.517(8)$ \\
\hline $\mathrm{C} 3-\mathrm{C} 4$ & $1.401(6)$ & $\mathrm{C} 18-\mathrm{C} 20$ & $1.503(11)$ \\
\hline $\mathrm{C} 3-\mathrm{C} 8$ & $1.502(5)$ & $\mathrm{C} 19-\mathrm{H} 19 \mathrm{~A}$ & 0.9800 \\
\hline $\mathrm{C} 4-\mathrm{H} 4$ & 0.9500 & С19-Н19B & 0.9800 \\
\hline $\mathrm{C} 4-\mathrm{C} 5$ & $1.393(6)$ & C19- H19C & 0.9800 \\
\hline $\mathrm{C} 5-\mathrm{C} 6$ & $1.401(6)$ & $\mathrm{C} 20-\mathrm{H} 20 \mathrm{~A}$ & 0.9800 \\
\hline $\mathrm{C} 5-\mathrm{O} 11$ & $1.373(5)$ & $\mathrm{C} 20-\mathrm{H} 20 \mathrm{~B}$ & 0.9800 \\
\hline $\mathrm{C} 6-\mathrm{C} 7$ & $1.393(5)$ & $\mathrm{C} 20-\mathrm{H} 20 \mathrm{C}$ & 0.9800 \\
\hline $\mathrm{C} 6-\mathrm{O} 13$ & $1.390(5)$ & $\mathrm{N} 22-\mathrm{C} 23$ & $1.355(5)$ \\
\hline $\mathrm{C} 7-\mathrm{H} 7$ & 0.9500 & $\mathrm{~N} 22-\mathrm{C} 27$ & $1.346(5)$ \\
\hline $\mathrm{C} 8-\mathrm{H} 8 \mathrm{~A}$ & 0.9900 & $\mathrm{C} 23-\mathrm{H} 23$ & 0.9500 \\
\hline $\mathrm{C} 8-\mathrm{H} 8 \mathrm{~B}$ & 0.9900 & $\mathrm{C} 23-\mathrm{C} 24$ & $1.379(6)$ \\
\hline $\mathrm{C} 8-\mathrm{C} 9$ & $1.523(6)$ & $\mathrm{C} 24-\mathrm{H} 24$ & 0.9500 \\
\hline $\mathrm{C} 9-\mathrm{H} 9$ & 0.9500 & $\mathrm{C} 24-\mathrm{C} 25$ & $1.396(5)$ \\
\hline $\mathrm{C} 9-\mathrm{C} 10$ & $1.376(6)$ & $\mathrm{C} 25-\mathrm{C} 26$ & $1.391(5)$ \\
\hline $\mathrm{C} 10-\mathrm{H} 10 \mathrm{~A}$ & 0.9500 & $\mathrm{C} 25-\mathrm{C} 28$ & $1.504(5)$ \\
\hline $\mathrm{C} 10-\mathrm{H} 10 \mathrm{~B}$ & 0.9500 & $\mathrm{C} 26-\mathrm{H} 26$ & 0.9500 \\
\hline $\mathrm{O} 11-\mathrm{C} 12$ & $1.431(5)$ & $\mathrm{C} 26-\mathrm{C} 27$ & $1.385(5)$ \\
\hline $\mathrm{C} 12-\mathrm{H} 12 \mathrm{~A}$ & 0.9800 & $\mathrm{C} 27-\mathrm{H} 27$ & 0.9500 \\
\hline C12-H12B & 0.9800 & $\mathrm{C} 28-\mathrm{O} 29$ & $1.206(5)$ \\
\hline $\mathrm{C} 12-\mathrm{H} 12 \mathrm{C}$ & 0.9800 & $\mathrm{C} 28-\mathrm{O} 30$ & $1.318(5)$ \\
\hline $\mathrm{O} 13-\mathrm{C} 14$ & $1.424(5)$ & $\mathrm{O} 30-\mathrm{H} 30$ & 0.8400 \\
\hline $\mathrm{C} 14-\mathrm{H} 14 \mathrm{~A}$ & 0.9900 & & \\
\hline
\end{tabular}




\begin{tabular}{|c|c|c|c|}
\hline $\mathrm{C} 2-\mathrm{Pt} 1-\mathrm{C} 9$ & $81.33(16)$ & $\mathrm{H} 12 \mathrm{~B}-\mathrm{C} 12-\mathrm{H} 12 \mathrm{C}$ & 109.5 \\
\hline $\mathrm{C} 2-\mathrm{Pt} 1-\mathrm{C} 10$ & $87.62(17)$ & $\mathrm{C} 6-\mathrm{O} 13-\mathrm{C} 14$ & $118.0(3)$ \\
\hline $\mathrm{C} 2-\mathrm{Pt} 1-\mathrm{Cl} 21$ & $93.86(12)$ & $\mathrm{O} 13-\mathrm{C} 14-\mathrm{H} 14 \mathrm{~A}$ & 109.1 \\
\hline $\mathrm{C} 2-\mathrm{Pt} 1-\mathrm{N} 22$ & $176.68(13)$ & $\mathrm{O} 13-\mathrm{C} 14-\mathrm{H} 14 \mathrm{~B}$ & 109.1 \\
\hline $\mathrm{C} 9-\mathrm{Pt} 1-\mathrm{Cl} 21$ & $163.54(12)$ & $\mathrm{O} 13-\mathrm{C} 14-\mathrm{C} 15$ & $112.5(3)$ \\
\hline $\mathrm{C} 9-\mathrm{Pt} 1-\mathrm{N} 22$ & $95.48(14)$ & $\mathrm{H} 14 \mathrm{~A}-\mathrm{C} 14-\mathrm{H} 14 \mathrm{~B}$ & 107.8 \\
\hline $\mathrm{C} 10-\mathrm{Pt} 1-\mathrm{C} 9$ & $37.64(16)$ & $\mathrm{C} 15-\mathrm{C} 14-\mathrm{H} 14 \mathrm{~A}$ & 109.1 \\
\hline $\mathrm{C} 10-\mathrm{Pt} 1-\mathrm{Cl} 21$ & $158.51(13)$ & $\mathrm{C} 15-\mathrm{C} 14-\mathrm{H} 14 \mathrm{~B}$ & 109.1 \\
\hline $\mathrm{C} 10-\mathrm{Pt} 1-\mathrm{N} 22$ & $90.39(15)$ & $\mathrm{O} 16-\mathrm{C} 15-\mathrm{C} 14$ & $126.1(4)$ \\
\hline $\mathrm{N} 22-\mathrm{Pt} 1-\mathrm{Cl} 21$ & $88.97(9)$ & $\mathrm{O} 16-\mathrm{C} 15-\mathrm{O} 17$ & $125.2(5)$ \\
\hline $\mathrm{C} 3-\mathrm{C} 2-\mathrm{Pt} 1$ & $115.9(3)$ & $\mathrm{O} 17-\mathrm{C} 15-\mathrm{C} 14$ & $108.7(4)$ \\
\hline $\mathrm{C} 3-\mathrm{C} 2-\mathrm{C} 7$ & $118.8(4)$ & $\mathrm{C} 15-\mathrm{O} 17-\mathrm{C} 18$ & $117.4(4)$ \\
\hline $\mathrm{C} 7-\mathrm{C} 2-\mathrm{Pt} 1$ & $125.3(3)$ & $\mathrm{O} 17-\mathrm{C} 18-\mathrm{H} 18$ & 109.1 \\
\hline $\mathrm{C} 2-\mathrm{C} 3-\mathrm{C} 4$ & $121.2(4)$ & $\mathrm{O} 17-\mathrm{C} 18-\mathrm{C} 19$ & $105.4(5)$ \\
\hline $\mathrm{C} 2-\mathrm{C} 3-\mathrm{C} 8$ & $117.6(4)$ & $\mathrm{O} 17-\mathrm{C} 18-\mathrm{C} 20$ & $108.8(5)$ \\
\hline $\mathrm{C} 4-\mathrm{C} 3-\mathrm{C} 8$ & $121.0(4)$ & $\mathrm{C} 19-\mathrm{C} 18-\mathrm{H} 18$ & 109.1 \\
\hline $\mathrm{C} 3-\mathrm{C} 4-\mathrm{H} 4$ & 120.2 & $\mathrm{C} 20-\mathrm{C} 18-\mathrm{H} 18$ & 109.1 \\
\hline $\mathrm{C} 5-\mathrm{C} 4-\mathrm{C} 3$ & $119.7(4)$ & $\mathrm{C} 20-\mathrm{C} 18-\mathrm{C} 19$ & $115.0(6)$ \\
\hline $\mathrm{C} 5-\mathrm{C} 4-\mathrm{H} 4$ & 120.2 & $\mathrm{C} 18-\mathrm{C} 19-\mathrm{H} 19 \mathrm{~A}$ & 109.5 \\
\hline $\mathrm{C} 4-\mathrm{C} 5-\mathrm{C} 6$ & $119.5(4)$ & $\mathrm{C} 18-\mathrm{C} 19-\mathrm{H} 19 \mathrm{~B}$ & 109.5 \\
\hline $\mathrm{O} 11-\mathrm{C} 5-\mathrm{C} 4$ & $125.2(4)$ & $\mathrm{C} 18-\mathrm{C} 19-\mathrm{H} 19 \mathrm{C}$ & 109.5 \\
\hline $\mathrm{O} 11-\mathrm{C} 5-\mathrm{C} 6$ & $115.2(4)$ & $\mathrm{H} 19 \mathrm{~A}-\mathrm{C} 19-\mathrm{H} 19 \mathrm{~B}$ & 109.5 \\
\hline $\mathrm{C} 7-\mathrm{C} 6-\mathrm{C} 5$ & $120.8(4)$ & $\mathrm{H} 19 \mathrm{~A}-\mathrm{C} 19-\mathrm{H} 19 \mathrm{C}$ & 109.5 \\
\hline $\mathrm{O} 13-\mathrm{C} 6-\mathrm{C} 5$ & $113.7(3)$ & $\mathrm{H} 19 \mathrm{~B}-\mathrm{C} 19-\mathrm{H} 19 \mathrm{C}$ & 109.5 \\
\hline $\mathrm{O} 13-\mathrm{C} 6-\mathrm{C} 7$ & $125.6(4)$ & $\mathrm{C} 18-\mathrm{C} 20-\mathrm{H} 20 \mathrm{~A}$ & 109.5 \\
\hline $\mathrm{C} 2-\mathrm{C} 7-\mathrm{H} 7$ & 120.0 & $\mathrm{C} 18-\mathrm{C} 20-\mathrm{H} 20 \mathrm{~B}$ & 109.5 \\
\hline $\mathrm{C} 6-\mathrm{C} 7-\mathrm{C} 2$ & $120.0(4)$ & $\mathrm{C} 18-\mathrm{C} 20-\mathrm{H} 20 \mathrm{C}$ & 109.5 \\
\hline $\mathrm{C} 6-\mathrm{C} 7-\mathrm{H} 7$ & 120.0 & $\mathrm{H} 20 \mathrm{~A}-\mathrm{C} 20-\mathrm{H} 20 \mathrm{~B}$ & 109.5 \\
\hline $\mathrm{C} 3-\mathrm{C} 8-\mathrm{H} 8 \mathrm{~A}$ & 109.7 & $\mathrm{H} 20 \mathrm{~A}-\mathrm{C} 20-\mathrm{H} 20 \mathrm{C}$ & 109.5 \\
\hline $\mathrm{C} 3-\mathrm{C} 8-\mathrm{H} 8 \mathrm{~B}$ & 109.7 & $\mathrm{H} 20 \mathrm{~B}-\mathrm{C} 20-\mathrm{H} 20 \mathrm{C}$ & 109.5 \\
\hline $\mathrm{C} 3-\mathrm{C} 8-\mathrm{C} 9$ & $109.9(3)$ & $\mathrm{C} 23-\mathrm{N} 22-\mathrm{Pt} 1$ & $121.5(3)$ \\
\hline $\mathrm{H} 8 \mathrm{~A}-\mathrm{C} 8-\mathrm{H} 8 \mathrm{~B}$ & 108.2 & $\mathrm{C} 27-\mathrm{N} 22-\mathrm{Pt} 1$ & $120.7(3)$ \\
\hline $\mathrm{C} 9-\mathrm{C} 8-\mathrm{H} 8 \mathrm{~A}$ & 109.7 & $\mathrm{C} 27-\mathrm{N} 22-\mathrm{C} 23$ & $117.8(3)$ \\
\hline $\mathrm{C} 9-\mathrm{C} 8-\mathrm{H} 8 \mathrm{~B}$ & 109.7 & $\mathrm{~N} 22-\mathrm{C} 23-\mathrm{H} 23$ & 118.8 \\
\hline $\mathrm{Pt} 1-\mathrm{C} 9-\mathrm{H} 9$ & 90.7 & $\mathrm{~N} 22-\mathrm{C} 23-\mathrm{C} 24$ & $122.4(4)$ \\
\hline $\mathrm{C} 8-\mathrm{C} 9-\mathrm{Pt} 1$ & $109.2(3)$ & $\mathrm{C} 24-\mathrm{C} 23-\mathrm{H} 23$ & 118.8 \\
\hline $\mathrm{C} 8-\mathrm{C} 9-\mathrm{H} 9$ & 119.1 & $\mathrm{C} 23-\mathrm{C} 24-\mathrm{H} 24$ & 120.4 \\
\hline $\mathrm{C} 10-\mathrm{C} 9-\mathrm{Pt} 1$ & $70.1(2)$ & $\mathrm{C} 23-\mathrm{C} 24-\mathrm{C} 25$ & $119.3(4)$ \\
\hline $\mathrm{C} 10-\mathrm{C} 9-\mathrm{C} 8$ & $121.8(4)$ & $\mathrm{C} 25-\mathrm{C} 24-\mathrm{H} 24$ & 120.4 \\
\hline $\mathrm{C} 10-\mathrm{C} 9-\mathrm{H} 9$ & 119.1 & $\mathrm{C} 24-\mathrm{C} 25-\mathrm{C} 28$ & $120.4(4)$ \\
\hline $\mathrm{Pt} 1-\mathrm{C} 10-\mathrm{H} 10 \mathrm{~A}$ & 107.8 & $\mathrm{C} 26-\mathrm{C} 25-\mathrm{C} 24$ & $118.6(4)$ \\
\hline $\mathrm{Pt} 1-\mathrm{C} 10-\mathrm{H} 10 \mathrm{~B}$ & 89.9 & $\mathrm{C} 26-\mathrm{C} 25-\mathrm{C} 28$ & $120.8(4)$ \\
\hline $\mathrm{C} 9-\mathrm{C} 10-\mathrm{Pt} 1$ & $72.3(2)$ & $\mathrm{C} 25-\mathrm{C} 26-\mathrm{H} 26$ & 120.7 \\
\hline $\mathrm{C} 9-\mathrm{C} 10-\mathrm{H} 10 \mathrm{~A}$ & 120.0 & $\mathrm{C} 27-\mathrm{C} 26-\mathrm{C} 25$ & $118.6(4)$ \\
\hline $\mathrm{C} 9-\mathrm{C} 10-\mathrm{H} 10 \mathrm{~B}$ & 120.0 & $\mathrm{C} 27-\mathrm{C} 26-\mathrm{H} 26$ & 120.7 \\
\hline $\mathrm{H} 10 \mathrm{~A}-\mathrm{C} 10-\mathrm{H} 10 \mathrm{~B}$ & 120.0 & $\mathrm{~N} 22-\mathrm{C} 27-\mathrm{C} 26$ & $123.2(4)$ \\
\hline $\mathrm{C} 5-\mathrm{O} 11-\mathrm{C} 12$ & $117.9(3)$ & $\mathrm{N} 22-\mathrm{C} 27-\mathrm{H} 27$ & 118.4 \\
\hline $\mathrm{O} 11-\mathrm{C} 12-\mathrm{H} 12 \mathrm{~A}$ & 109.5 & $\mathrm{C} 26-\mathrm{C} 27-\mathrm{H} 27$ & 118.4 \\
\hline
\end{tabular}




$\mathrm{O} 11-\mathrm{C} 12-\mathrm{H} 12 \mathrm{~B}$
$\mathrm{O} 11-\mathrm{C} 12-\mathrm{H} 12 \mathrm{C}$
$\mathrm{H} 12 \mathrm{~A}-\mathrm{C} 12-\mathrm{H} 12 \mathrm{~B}$
$\mathrm{H} 12 \mathrm{~A}-\mathrm{C} 12-\mathrm{H} 12 \mathrm{C}$
$\mathrm{Pt} 1-\mathrm{C} 2-\mathrm{C} 3-\mathrm{C} 4$
$\mathrm{Pt} 1-\mathrm{C} 2-\mathrm{C} 3-\mathrm{C} 8$
$\mathrm{Pt} 1-\mathrm{C} 2-\mathrm{C} 7-\mathrm{C} 6$
$\mathrm{Pt} 1-\mathrm{N} 22-\mathrm{C} 23-\mathrm{C} 24$
$\mathrm{Pt} 1-\mathrm{N} 22-\mathrm{C} 27-\mathrm{C} 26$
$\mathrm{C} 2-\mathrm{C} 3-\mathrm{C} 4-\mathrm{C} 5$
$\mathrm{C} 2-\mathrm{C} 3-\mathrm{C} 8-\mathrm{C} 9$
$\mathrm{C} 3-\mathrm{C} 2-\mathrm{C} 7-\mathrm{C} 6$
$\mathrm{C} 3-\mathrm{C} 4-\mathrm{C} 5-\mathrm{C} 6$
$\mathrm{C} 3-\mathrm{C} 4-\mathrm{C} 5-\mathrm{O} 11$
$\mathrm{C} 3-\mathrm{C} 8-\mathrm{C} 9-\mathrm{P} 1$
$\mathrm{C} 3-\mathrm{C} 8-\mathrm{C} 9-\mathrm{C} 10$
$\mathrm{C} 4-\mathrm{C} 3-\mathrm{C} 8-\mathrm{C} 9$
C4-C5-C6-C7
C4-C5-C6-O13
C4-C5-O11-C12
C5-C6-C7-C2
C5-C6-O13-C14
C6-C5-O11-C12
C6-O13-C14-C15
C7-C2-C3-C4
C7-C2-C3-C8
C7-C6-O13-C14

$\mathrm{O} 11-\mathrm{C} 12-\mathrm{H} 12 \mathrm{~B}$

$\mathrm{H} 12 \mathrm{~A}-\mathrm{C} 12-\mathrm{H} 12 \mathrm{~B}$

$\mathrm{H} 12 \mathrm{~A}-\mathrm{C} 12-\mathrm{H} 12 \mathrm{C}$

$\mathrm{Pt} 1-\mathrm{C} 2-\mathrm{C} 3-\mathrm{C} 4$

$\mathrm{P} 11-\mathrm{C} 2-\mathrm{C} 7-\mathrm{C} 6$

Pt1-N22-C23-C24

$\mathrm{C} 2-\mathrm{C} 3-\mathrm{C} 4-\mathrm{C} 5$

$\mathrm{C} 2-\mathrm{C} 3-\mathrm{C} 8-\mathrm{C} 9$

$\mathrm{C} 3-\mathrm{C} 2-\mathrm{C} 7-\mathrm{C} 6$

$\mathrm{C} 3-\mathrm{C} 8-\mathrm{C} 9-\mathrm{Pt} 1$

$\mathrm{C} 3-\mathrm{C} 8-\mathrm{C} 9-\mathrm{C} 10$

$\mathrm{C} 4-\mathrm{C} 3-\mathrm{C} 8-\mathrm{C} 9$

$\mathrm{C} 4-\mathrm{C} 5-\mathrm{C} 6-\mathrm{C} 7$

$\mathrm{C} 4-\mathrm{C} 5-\mathrm{C} 6-\mathrm{O} 13$

$\mathrm{C} 4-\mathrm{C} 5-\mathrm{O} 11-\mathrm{C} 12$

$\mathrm{C}+\mathrm{C} 6-\mathrm{O} 13-\mathrm{C} 14$
109.5

109.5

109.5

109.5

$177.9(3)$

$1.8(5)$

$-177.7(3)$

$179.1(3)$

$178.8(3)$

$-0.1(6)$

$-19.4(5)$

$0.0(6)$

$0.1(6)$

$178.1(4)$

$26.1(4)$

$-51.9(5)$

$164.5(4)$

$-0.1(6)$

$-179.5(4)$

$1.1(6)$

$0.1(6)$

$176.9(4)$

$179.1(4)$

-73.7 (4)

$0.0(6)$

$-176.1(4)$

$-2.4(6)$
$\mathrm{O} 29-\mathrm{C} 28-\mathrm{C} 25$
$\mathrm{O} 29-\mathrm{C} 28-\mathrm{O} 30$
$\mathrm{O} 30-\mathrm{C} 28-\mathrm{C} 25$
$\mathrm{C} 28-\mathrm{O} 30-\mathrm{H} 30$

$\mathrm{C} 8-\mathrm{C} 3-\mathrm{C} 4-\mathrm{C} 5$

$\mathrm{C} 8-\mathrm{C} 9-\mathrm{C} 10-\mathrm{Pt} 1$

$\mathrm{O} 11-\mathrm{C} 5-\mathrm{C} 6-\mathrm{C} 7$

$\mathrm{O} 11-\mathrm{C} 5-\mathrm{C} 6-\mathrm{O} 13$

$\mathrm{O} 13-\mathrm{C} 6-\mathrm{C} 7-\mathrm{C} 2$

$\mathrm{O} 13-\mathrm{C} 14-\mathrm{C} 15-\mathrm{O} 16$

$\mathrm{O} 13-\mathrm{C} 14-\mathrm{C} 15-\mathrm{O} 17$

$\mathrm{C} 14-\mathrm{C} 15-\mathrm{O} 17-\mathrm{C} 18$

C15-O17-C18-C19

$\mathrm{C} 15-\mathrm{O} 17-\mathrm{C} 18-\mathrm{C} 20$

$\mathrm{O} 16-\mathrm{C} 15-\mathrm{O} 17-\mathrm{C} 18$

$\mathrm{N} 22-\mathrm{C} 23-\mathrm{C} 24-\mathrm{C} 25$

$\mathrm{C} 23-\mathrm{N} 22-\mathrm{C} 27-\mathrm{C} 26$

$\mathrm{C} 23-\mathrm{C} 24-\mathrm{C} 25-\mathrm{C} 26$

$\mathrm{C} 23-\mathrm{C} 24-\mathrm{C} 25-\mathrm{C} 28$

$\mathrm{C} 24-\mathrm{C} 25-\mathrm{C} 26-\mathrm{C} 27$

$\mathrm{C} 24-\mathrm{C} 25-\mathrm{C} 28-\mathrm{O} 29$

$\mathrm{C} 24-\mathrm{C} 25-\mathrm{C} 28-\mathrm{O} 30$

$\mathrm{C} 25-\mathrm{C} 26-\mathrm{C} 27-\mathrm{N} 22$

$\mathrm{C} 26-\mathrm{C} 25-\mathrm{C} 28-\mathrm{O} 29$

$\mathrm{C} 26-\mathrm{C} 25-\mathrm{C} 28-\mathrm{O} 30$

$\mathrm{C} 27-\mathrm{N} 22-\mathrm{C} 23-\mathrm{C} 24$

$\mathrm{C} 28-\mathrm{C} 25-\mathrm{C} 26-\mathrm{C} 27$
122.4 (4)

125.7 (4)

$112.0(3)$

109.5

$175.9(4)$

100.7 (4)

$-178.3(4)$

$2.4(5)$

179.3 (4)

$10.9(6)$

$-166.5(4)$

$-179.9(5)$

$-146.0(5)$

$90.2(6)$

$2.7(8)$

$2.6(7)$

$-0.2(6)$

$-1.2(6)$

$-176.6(4)$

$-0.8(6)$

$21.8(6)$

$-159.1(4)$

$1.6(6)$

-153.5 (4)

$25.6(6)$

$-1.9(6)$

$174.6(4)$

Hydrogen-bond geometry $\left(A,{ }^{\circ}\right)$

\begin{tabular}{lllll}
\hline$D-\mathrm{H} \cdots A$ & $D-\mathrm{H}$ & $\mathrm{H} \cdots A$ & $D \cdots A$ & $D-\mathrm{H} \cdots A$ \\
\hline $\mathrm{O} 30-\mathrm{H} 30 \cdots \mathrm{O} 13^{\mathrm{i}}$ & 0.84 & 2.10 & $2.932(4)$ & 170 \\
$\mathrm{C} 10-\mathrm{H} 10 A \cdots \mathrm{O} 16^{\mathrm{ii}}$ & 0.95 & 2.41 & $3.317(5)$ & 159 \\
$\mathrm{C} 12-\mathrm{H} 12 A \cdots \mathrm{O} 16^{\mathrm{iii}}$ & 0.98 & 2.51 & $3.415(5)$ & 154 \\
$\mathrm{C} 14-\mathrm{H} 14 A \cdots \mathrm{O} 29^{\mathrm{iv}}$ & 0.99 & 2.46 & $3.268(5)$ & 139 \\
$\mathrm{C} 14-\mathrm{H} 14 B \cdots \mathrm{O} 29^{\mathrm{v}}$ & 0.99 & 2.46 & $3.178(5)$ & 129 \\
$\mathrm{C} 26-\mathrm{H} 26 \cdots \mathrm{O} 16^{\mathrm{vi}}$ & 0.95 & 2.43 & $3.336(5)$ & 159 \\
\hline
\end{tabular}

Symmetry codes: (i) $x-1, y, z-1$; (ii) $x-1, y, z$; (iii) $-x+1,-y+1,-z+1$; (iv) $x+1, y, z+1$; (v) $-x,-y+2,-z$; (vi) $-x,-y+1,-z$. 